\title{
Social Dynamics Models with Time-Varying Influence
}

\author{
Sean McQuade ${ }^{*}$, Benedetto Piccoli ${ }^{*}$, and Nastassia Pouradier Duteil ${ }^{\dagger}$ \\ * Department of Mathematical Sciences, Rutgers University, Camden, \\ NJ 08102, USA \\ †Sorbonne Université, Inria, Université Paris-Diderot SPC, CNRS, \\ Laboratoire Jacques-Louis Lions, Paris, France
}

April 4, 2019

\begin{abstract}
This paper introduces an augmented model for first-order opinion dynamics, in which a weight of influence is attributed to each agent. Each agent's influence on another agent's opinion is then proportional not only to the classical interaction function, but also to its weight. The weights evolve in time and their equations are coupled with the opinions' evolution. We show that the well-known conditions for convergence to consensus can be generalized to this framework. In the case of interaction functions with bounded support, we show that constant weights lead to clustering with conditions similar to those of the classical model.

Four specific models are designed by prescribing a specific weight dynamics, then the convergence of the opinions and the evolution of the weights for each of them is studied. We prove the existence of different long-term behaviors, such as emergence of a single leader and emergence of two co-leaders. The we illustrate them via numerical simulations. Lastly, a statistical analysis is provided for the speed of convergence to consensus and for the clustering behavior of each model, together with a comparison to the classical opinion dynamics with constant equal weights.
\end{abstract}

\section{Introduction}

The applied mathematics community has been very active in recent years in studying the dynamics of large systems of active agents, also called social dynamics. The interest stems out of the many different problems which can be modeled within this framework, such as animal groups $[4,5,8,13,19]$. In most applications, the interest is in large groups, e.g. bird flocks with thousands of members [3], and the term social refers to the nature of interaction rules. Moreover, one of the key phenomena is self-organization [6], i.e. the spontaneous emergence of well organized group patterns which results from the local interaction rules without any single group member 
perceiving the global picture. Self-organization patterns include consensus, alignment, clustering, or the less studied dancing equilibrium [2,7].

More specifically, in opinion dynamics, various models were proposed with different interaction rules. The first contributions date back to the 50's, see [12, 14], then linear models were proposed by De Groot [9] and Lehrer [22]. More recent models include the celebrated bounded-confidence model by Hegselmann and Krause (briefly HK model) [16] and others, see [10, 15, 20, 21]. The HK model produces clustering by limiting the distance at which agents interact and [23] showed that heterophilious dynamics enhances consensus. We refer the reader to [1] and references therein for a complete discussion. In such models an agent interacts with the others depending on their opinion distance, possibly their topological position in the interaction network, and possibly some predetermined influence weights. Here we consider an augmented framework in which, in addition to the pairwise interaction function, a time-varying weight of influence is attributed to each agent. This weight allows us to model a social hierarchy within the group, where the most influential agents (i.e. those with the larger weights) have a much larger impact on the behavior of the group. The dynamics of the weights can be prescribed in a number of ways. We present different models coupling the opinions' dynamics and the weights' dynamics. We refer to [26] for a recent model with time-evolving masses for pedestrian dynamics.

We propose four different models for the weights' evolution. For all models we impose the conservation of the sum of the weights, so that the weights simply shift between agents. In Model 1, agents gain mass if they attract the other agents more than they are attracted in pairwise interactions. This is achieved by looking at the midpoint dynamics. In Model 2, an agent gains mass if its stance or opinion is well received by many others. An example could model a politician using a wholly popular idea to gain influence. Many students and young voters may give support to free college in the United States which increases the influence of the agent with this idea. In Model 3 , an agent gains mass by attracting other influential individuals. This model is appropriate for modeling opinions in the situation where very influential people appear to support opinions publicly. An example is when a musician, movie star, or popular artist supports a political candidate. The political candidate may gain influence because they appear to attract other influential individuals. Lastly, Model 4 represents a continuous version of Model 3, where we replace the set of most influential agents with a continuously varying coefficient.

We first address the problem of existence of solutions. Our models are smooth in the space variable but present natural discontinuities in the weight dynamics. Thus we consider solutions in the Filippov sense and prove that the space trajectory is always unique. This allows to prove that agents never coalesce in finite time and, for Models 1 and 2, if we start with some agents in the same position we can replace them by a single one whose weight is the sum of the weights.

We then prove some general properties of the system: the maximal norm of opinions is contracting as well as the convex hull of opinions. We also provide estimates on the diameter dynamics in terms of the interaction functional. We next focus on specific properties of the interaction function. If the interaction does not vanish for large distances, then consensus is always achieved exponentially fast in time. For the boundedconfidence case we prove that clustering occurs using an appropriate energy functional. 
We then deal with heterophilious dynamics, in the specific case of a constant interaction function. In this simplified case, we highlight the main characteristics and constitutive differences in the large-time dynamics of our four models. For Model 1, the weights never vanish and have an a-priori lower bound. Therefore, no agent looses completely its weight (not even asymptotically) and no agent gains all the weight. On the contrary, for Model 2, a single agent will asymptotically gain all the weight and such agent can be identified a priori. Finally, for Models 3 and 4, equilibria exhibit two agents sharing all the weight and we conjecture that this are also the asymptotic states.

Then we turn our attention to numerical simulations. We show the behavior of all models with various agents starting from random positions and with random weights. We also measure the speed of convergence to consensus (when it is achieved) and show that the weight dynamics tend to facilitate consensus and increase the convergence speed for some of the proposed models. We then compare the clustering behavior of the four models in the case of an interaction function with bounded support. Lastly, we provide some conclusions and future directions.

\section{General results}

\subsection{Notations and model definitions}

Let $\left(x_{i}\right)_{i \in\{1, \cdots, N\}} \in\left(\mathbb{R}^{d}\right)^{N}$ represent the opinions (or positions) of a group of $N$ agents. A general opinion dynamics model, as introduced by Hegselmann and Krause in [16], can be written as:

$$
\left\{\begin{array}{l}
\dot{x}_{i}(t)=\frac{1}{N} \sum_{j=1}^{N} a\left(\left\|x_{i}(t)-x_{j}(t)\right\|\right)\left(x_{j}(t)-x_{i}(t)\right), \quad i \in\{1, \cdots, N\}, \\
x_{i}(0)=x_{i}^{0}
\end{array}\right.
$$

where $a(\cdot)$ represents the interaction function. This general system models the dynamics of a group of agents in which each agent $j$ 's influence on another individual $i$ depends on the distance between them and on the position of $j$ with respect to $i$.

We extend this model by attributing to each agent a weight of influence $m_{i} \in \mathbb{R}^{+}$, which evolves in time due to its own dynamics (note that we will also refer to it as the agent's mass). Let $M:=\sum_{i=1}^{N} m_{i}$ be the total weight (or mass) of the system. A general form of this augmented model will be written as:

$$
\left\{\begin{array}{l}
\dot{x}_{i}(t)=\frac{1}{M} \sum_{j=1}^{N} m_{j}(t) a\left(\left\|x_{i}(t)-x_{j}(t)\right\|\right)\left(x_{j}(t)-x_{i}(t)\right), \\
\dot{m}_{i}(t)=\Psi\left(m(t), x(t), m_{i}(t), x_{i}(t)\right), \\
x_{i}(0)=x_{i}^{0}, \quad m_{i}(0)=m_{i}^{0},
\end{array} i \in\{1, \cdots, N\},\right.
$$

where we denoted by $m$ the vector of $N$ weights $m=\left(m_{i}\right)_{i \in\{1, \cdots, N\}}$ and by $x$ the $\mathrm{N}$ opinions $x=\left(x_{i}\right)_{i \in\{1, \cdots, N\}}$. In this new approach, an agent $j$ 's influence on $i$ no longer depends solely on the vector $x_{j}-x_{i}$, but also on the weight $m_{j}$. This timevarying weight dynamics will be generally prescribed by a function $\Psi$ depending on 
the group's opinions $\left(x_{i}\right)_{i \in\{1, \cdots, N\}}$ and weights of influence $\left(m_{i}\right)_{i \in\{1, \cdots, N\}}$. From here onward, for more simplicity we will denote by $\psi_{i}$ the function $\Psi$ evaluated at $x_{i}$ and $m_{i}$, i.e. $\psi_{i}(m, x):=\Psi\left(m, x, m_{i}, x_{i}\right)$. We rewrite system (2) in the condensed form:

$$
\left\{\begin{array}{l}
\dot{x}_{i}(t)=X_{i}(x(t), m(t)), \\
\dot{m}_{i}(t)=\psi_{i}(x(t), m(t)), \\
x_{i}(0)=x_{i}^{0}, \quad m_{i}(0)=m_{i}^{0},
\end{array} \quad i \in\{1, \cdots, N\}\right.
$$

where we denoted by $X_{i}:\left(\mathbb{R}^{d}\right)^{N} \times \mathbb{R}^{N} \rightarrow\left(\mathbb{R}^{d}\right)^{N}$ the function

$$
X_{i}:(x, m) \mapsto \frac{1}{M} \sum_{j=1}^{N} m_{j} a\left(\left\|x_{i}-x_{j}\right\|\right)\left(x_{j}-x_{i}\right) .
$$

Furthermore, we assume that for each $i \in\{1, \cdots, N\}$, there exists a function $\bar{\psi}_{i}$ such that

$$
\forall(x, m) \in\left(\mathbb{R}^{d}\right)^{N} \times \mathbb{R}^{N}, \quad \psi_{i}(x, m)=m_{i} \bar{\psi}_{i}(x, m) .
$$

We will also use the notations

$$
X:=\left(X_{i}\right)_{i \in\{1, \cdots, N\}}, \quad \psi:=\left(\psi_{i}\right)_{i \in\{1, \cdots, N\}}, \quad \text { and } \quad \bar{\psi}:=\left(\bar{\psi}_{i}\right)_{i \in\{1, \cdots, N\}}
$$

and from here onward we assume the following:

Hypothesis 1. The function $s \mapsto a(\|s\|) s$ is locally Lipschitz in $\mathbb{R}^{d}$, and the function $\psi$ is locally bounded in $\left(\mathbb{R}^{d}\right)^{N} \times \mathbb{R}^{N}$.

Remark 1. The fact that the mass dynamics satisfy (5) together with Hypothesis 1 implies that non-negative weights remain non-negative at all time.

We also require the following condition on the weight dynamics:

Hypothesis 2. For all $(x, m) \in\left(\mathbb{R}^{d}\right)^{N} \times \mathbb{R}^{N}$,

$$
\sum_{i=1}^{N} \psi_{i}(x, m)=0
$$

This implies that the total weight $M$ of the group stays constant, so that the weight distribution shifts between agents while the sum of the weights remains unchanged. As a consequence, the weights satisfy $m \in[0, M]^{N}$, where $M=\sum_{i=1}^{N} m_{i}^{0}$.

From here onward, unless we specify otherwise, all results concern the general model with weights (3)-(4)-(5), together with Hypotheses 1 and 2.

We now present four specific models in which we prescribe the dynamics $\psi_{i}$ of the weights of influence. The models we build rely on giving a meaning to the following quantities:

- $x_{i}$, referred to as the opinion (or position) of agent $i$

- $m_{i}$, referred to as the weight (or mass) of agent $i$ 
- $M=\sum_{i=1}^{N} m_{i}^{0}$, referred to as the total mass of the system

- $a\left(\left\|x_{i}-x_{j}\right\|\right)\left\|x_{i}-x_{j}\right\|$, referred to as the positional influence of $i$ over $j$ (and symmetrically, of $j$ over $i$ )

- $p_{i}:=\sum_{j=1}^{N} a\left(\left\|x_{i}-x_{j}\right\|\right)\left\|x_{i}-x_{j}\right\|$, referred to as the total positional influence of $i$ over the group

- $e_{i j}:=m_{j} a\left(\left\|x_{i}-x_{j}\right\|\right)\left\|x_{i}-x_{j}\right\|$, referred to as the the influence of $j$ over $i$ (notice that $e_{i j}$ is obtained by multiplying the positional influence of $j$ over $i$ by the weight of $j$ )

- $I_{i}:=\operatorname{argmax}_{j \in\{1, \cdots, N\}} e_{i j}=\left\{j \in\{1, \cdots, N\} \mid \forall k \in\{1, \cdots, N\}, e_{i j} \geq e_{i k}\right\}$, representing the set of agents that have the largest influence on $i$

Model 1: Increasing weight by pairwise competition. In this first model, the dynamics of an agent's weight $m_{i}$ depend on the dynamics of the midpoints between $i$ and each other agent $j$ :

$$
\left\{\begin{array}{l}
\dot{x}_{i}=\frac{1}{M} \sum_{j=1}^{N} m_{j} a\left(\left\|x_{i}-x_{j}\right\|\right)\left(x_{j}-x_{i}\right) \\
\dot{m}_{i}=\frac{m_{i}}{M} \sum_{j=1}^{N} m_{j}\left\langle\frac{\dot{x}_{i}+\dot{x}_{j}}{2}, u_{j i}\right\rangle
\end{array} \quad i \in\{1, \cdots, N\}\right.
$$

where $u_{j i}$ is the vector defined by:

$$
u_{j i}= \begin{cases}\frac{x_{i}-x_{j}}{\left\|x_{i}-x_{j}\right\|} & \text { if } x_{i} \neq x_{j} \\ 0 & \text { if } x_{i}=x_{j}\end{cases}
$$

If the midpoint $\frac{x_{i}+x_{j}}{2}$ moves in the direction of $x_{i}$, i.e. $\left\langle\frac{\dot{x}_{i}+\dot{x}_{j}}{2}, \frac{x_{i}-x_{j}}{\left\|x_{i}-x_{j}\right\|}\right\rangle \geq 0$, then $i$ gains influence and $m_{i}$ increases, proportionally to the projection of the velocity of the midpoint on the direction $\frac{x_{i}-x_{j}}{\left\|x_{i}-x_{j}\right\|}$ and proportionally to $j$ 's own mass $m_{j}$. Notice that for compactness of notation, we expressed $\dot{m}_{i}$ in terms of $x, m$ and $\dot{x}$. However, by plugging the expressions of $\dot{x}_{i}$ and $\dot{x}_{j}$ in the second equation of (M1), we recover an equation of the form $\dot{m}_{i}=\psi_{i}(x, m)$, so we are in the framework of the general system (3)-(4)-(5).

Model 2: Increasing weight by positional influence. In this model, an agent's weight $m_{i}$ increases if the agent's positional influence $p_{i}$ is higher than the weighted average of the group's positional influences. We write:

$$
\left\{\begin{array}{l}
\dot{x}_{i}=\frac{1}{M} \sum_{j=1}^{N} m_{j} a\left(\left\|x_{i}-x_{j}\right\|\right)\left(x_{j}-x_{i}\right) \\
\dot{m}_{i}=\frac{m_{i}}{M}\left(\frac{p_{i}}{\frac{1}{M} \sum_{j=1}^{N} m_{j} p_{j}}-1\right)
\end{array} \quad i \in\{1, \cdots, N\},\right.
$$


where $p_{i}:=\sum_{j=1}^{N} a\left(\left\|x_{i}-x_{j}\right\|\right)\left\|x_{i}-x_{j}\right\|$ represents an agent's total positional influence on the group.

Model 3: Increasing weight by exerting the biggest influence on the most influential. In this model, $m_{i}$ will increase if the agents that $i$ influences the most are themselves influential. We give:

$$
\left\{\begin{array}{l}
\dot{x}_{i}=\frac{1}{M} \sum_{j=1}^{N} m_{j} a\left(\left\|x_{i}-x_{j}\right\|\right)\left(x_{j}-x_{i}\right) \\
\dot{m}_{i}=m_{i}\left(\sum_{j \mid i \in I_{j}} \frac{m_{j}}{\left|I_{j}\right|_{m}}-1\right)
\end{array} \quad i \in\{1, \cdots, N\}\right.
$$

where $\left|I_{j}\right|_{m}:=\sum_{k \in I_{j}} m_{k}$ represents the weight of the agents that influence $j$ the most. As defined earlier, $I_{j}=\operatorname{argmax}_{k \in\{1, \cdots, N\}} e_{j k}$ is the set of agents that influence $j$ the most, with $e_{j k}=m_{k} a\left(\left\|x_{j}-x_{k}\right\|\right)\left\|x_{j}-x_{k}\right\|$ representing the influence of $k$ over $j$. A sufficient condition for $m_{i}$ to increase is if there exists $j$ with a greater weight than $i$ (i.e. $m_{j}>m_{i}$ ) such that $i$ is the most influential agent on $j$, i.e. $I_{j}=\{i\}$. More generally, if $\# I_{j}=1$ for all $j \in\{1, \cdots, N\}$ (where \# denotes the cardinality of a set), $\dot{m}_{i}>0$ if and only if the sum of the weights of all the agents for whom agent $i$ is the most influential is greater than its own weight $m_{i}$. Indeed, in this case, $i \in I_{j}$ implies that $\left|I_{j}\right|_{m}=m_{i}$, and we get:

$$
\dot{m}_{i}>0 \Leftrightarrow \sum_{j \mid i \in I_{j}} \frac{m_{j}}{\left|I_{j}\right|_{m}}>1 \Leftrightarrow \sum_{j \mid i \in I_{j}} \frac{m_{j}}{m_{i}}>1 \Leftrightarrow \sum_{j \mid i \in I_{j}} m_{j}>m_{i} .
$$

Model 4: Increasing weight by influencing the most influential. This last model provides a "continuous" version of the topological model (M3):

$$
\left\{\begin{array}{l}
\dot{x}_{i}=\frac{1}{M} \sum_{j=1}^{N} m_{j} a\left(\left\|x_{i}-x_{j}\right\|\right)\left(x_{j}-x_{i}\right) \\
\dot{m}_{i}=m_{i}\left(\frac{1}{M} \sum_{j=1}^{N} m_{j} \frac{e_{j i}}{\frac{1}{M} \sum_{k=1}^{N} m_{k} e_{j k}}-1\right)
\end{array} \quad i \in\{1, \cdots, N\},\right.
$$

where $e_{j i}=m_{i} a\left(\left\|x_{i}-x_{j}\right\|\right)\left\|x_{i}-x_{j}\right\|$ represents the influence of agent $i$ on agent $j$. In this model, an agent $j$ contributes to increasing the weight of an agent $i$ proportionally to its own weight $m_{j}$ and to the strength of the influence of $i$ on $j$ relatively to the weighted average of all influences on $j$. As opposed to Model (M3), in the evolution of $m_{i}$ we consider not only the agents for which $i$ has the strongest influence, but all agents $j$, with a weight corresponding to how much $i$ counts in the total influence perceived by $j$.

\subsection{Existence and uniqueness of solutions}

We begin by establishing the existence of solutions to the general system (3)-(4)-(5). Let us first recall the definition of Filippov set-valued maps (see [11]): 
Definition 1. Let $\mathscr{P}(E)$ denote the collection of susbsets of the Euclidean space E. For a measurable vector field $X: E \rightarrow E$, the Filippov set-valued map $F[X]: E \rightarrow \mathscr{P}(E)$ is defined by:

$$
\forall x \in E, \quad F[X](x):=\bigcap_{\delta>0} \bigcap_{S \in \mathscr{P}(E), \mu(S)=0} \overline{\operatorname{co}}\{X(B(x, \delta) \backslash S)\},
$$

where $\overline{c o}\{\cdot\}$ denotes the convex closure, $\mu$ denotes the Lebesgue measure, $B(x, \delta)$ denotes the ball of center $x$ and radius $\delta$, and for all $S \in \mathscr{P}(E), X(S):=\{X(x) \mid x \in S\}$.

Proposition 1. Consider the differential system (3)-(4)-(5) and suppose that $s \mapsto$ $a(\|s\|) s \in \operatorname{Lip}_{\text {loc }}\left(\mathbb{R}^{d}\right)$ and $\psi \in L_{\mathrm{loc}}^{\infty}\left(\left(\mathbb{R}^{d}\right)^{N} \times \mathbb{R}^{N}\right)$. Then there exists a solution $(x, m)$ to system (3)-(4)-(5) in the sense of Filippov, i.e. an absolutely continuous map $(x, m):[0, T] \rightarrow\left(\mathbb{R}^{d}\right)^{N} \times \mathbb{R}^{N}$ such that for almost all $t \in[0, T]$,

$$
(\dot{x}, \dot{m})(t) \in F[(X, \psi)](x(t),(m(t)),
$$

where $F[(X, \psi)]$ denotes the Filippov set-valued map of $(X, \psi)$. Furthermore, for all solutions $(x, m)$ and $(y, p)$ satisfying $(x(0), m(0))=(y(0), q(0))$, if $m \equiv q$, then $x \equiv y$.

Proof. The existence of solutions is a direct application of the notion of Filippov solutions [11], since the local boundedness of $(X, \psi)$ implies the upper semi-continuity of $F[(X, \psi)]$. Uniqueness of solutions is not guaranteed since $(X, \psi)$ is not locally Lipschitz. However, for each solution $t \mapsto m(t)$, the first equation of system (3)-(4)-(5) can be rewritten as:

$$
\dot{x}_{i}=X_{i}(x, m(t)):=\tilde{X}_{i}(x, t)
$$

where $\tilde{X}:=\left(\tilde{X}_{i}\right)_{i \in\{1, \cdots, N\}}$ is locally Lipschitz with respect to the $\ell_{1}^{N}-\ell_{2}^{d}$ norm $x \mapsto$ $\sum_{i=1}^{N}\left\|x_{i}\right\|$, where $\|\cdot\|$ is the Euclidean norm of $\mathbb{R}^{d}$. Indeed, let $\delta>0$, and let $(x, y) \in$ $\left(\mathbb{R}^{d N}\right)^{2}$ such that $\sum_{i=1}^{N}\left\|x_{i}-y_{i}\right\| \leq \delta$. Then for all $(i, j) \in\{1, \cdots, N\}^{2}, \|\left(x_{j}-x_{i}\right)-$ $\left(y_{j}-y_{i}\right)\|\leq\|\left(x_{j}-y_{j}\right)\|+\|\left(x_{i}-y_{i}\right) \| \leq \delta$, so there exists $K \geq 0$ such that for each $i \in\{1, \cdots, N\}$,

$$
\begin{aligned}
\left\|\tilde{X}_{i}(t, x)-\tilde{X}_{i}(t, y)\right\| & =\left\|\frac{1}{M} \sum_{j=1}^{N} m_{j}\left[a\left(\left\|x_{i}-x_{j}\right\|\right)\left(x_{j}-x_{i}\right)-a\left(\left\|y_{i}-y_{j}\right\|\right)\left(y_{j}-y_{i}\right)\right]\right\| \\
& \leq \frac{1}{M} \sum_{j=1}^{N} m_{j} K\left\|\left(x_{j}-x_{i}\right)-\left(y_{j}-y_{i}\right)\right\| \\
& \leq \frac{K}{M} \sum_{j=1}^{N} m_{j}\left(\left\|x_{j}-y_{j}\right\|+\left\|x_{i}-x_{j}\right\|\right) \leq 2 K \sum_{j=1}^{N}\left\|x_{j}-y_{j}\right\| .
\end{aligned}
$$

Hence (7) admits a unique solution from each initial condition $x(0)=x_{0} \in\left(\mathbb{R}^{d}\right)^{N}$.

An important property follows from the uniqueness of $x$ given the weights $t \mapsto m(t)$ : if two agents are clustered at some time $T$, i.e. $x_{i}(T)=x_{j}(T)$, then they are necessarily clustered at all time before and after $T$. As a consequence, in one-dimension, the dynamics preserve the order. 
Corollary 1. Let $(x, m): \mathbb{R}^{+} \mapsto\left(\mathbb{R}^{d}\right)^{N} \times[0, M]^{N}$ denote a solution to system (2). If for some $T \geq 0, x_{i}(T)=x_{j}(T)$, then $x_{i}(t)=x_{j}(t)$ for all $t \in \mathbb{R}^{+}$. Hence, if $d=1$, if $x_{i}(0) \geq x_{j}(0)$, then $x_{i}(t) \geq x_{j}(t)$ for all $t \in \mathbb{R}^{+}$.

Proof. Suppose that for some $T \geq 0, x_{i}(T)=x_{j}(T)$. Then from the dynamics (2), one easily computes: $\dot{x}_{i}(T)=\dot{x}_{j}(T)$. From Proposition 1 , there is a unique solution $x$ for each $m$ satisfying (2), hence $x_{i}(t)=x_{j}(t)$ for all $t \geq T$ and $t \leq T$.

From here onward, we will suppose that at initial time, $x_{i}^{0} \neq x_{j}^{0}$ for all $(i, j) \in$ $\{1, \cdots, N\}^{2}$. In two of the specific models defined in Section 2.1, this can be done without loss of generality, as we show with the following:

Lemma 1. Let $t \mapsto(x(t), m(t)) \in \mathbb{R}^{d N} \times \mathbb{R}^{N}$ and $t \mapsto(y(t), q(t)) \in \mathbb{R}^{d(N-1)} \times \mathbb{R}^{N-1}$ be two solutions to system (M1) (respectively (M2)). If the initial conditions satisfy

$$
\left\{\begin{array}{l}
x_{1}^{0}=x_{N}^{0}=y_{1}^{0} \\
m_{1}^{0}+m_{N}^{0}=q_{1}^{0}
\end{array}\right.
$$

then $y_{1} \equiv x_{1} \equiv x_{N}, q_{1} \equiv m_{1}+m_{N}$, and for all $i \in\{2, \cdots, N-1\}, y_{i} \equiv x_{i}$ and $q_{i} \equiv m_{i}$.

Proof. First, from Corollary 1, it follows immediately that $x_{1} \equiv x_{N}$, independently of the weight dynamics. Secondly, let $\tilde{x}:=\left(x_{1}, \cdots, x_{N-1}\right)$ and $\tilde{m}:=\left(m_{1}+m_{N}, \cdots, m_{N-1}\right)$. We show that for each of the two models (M1) and (M2), $(\tilde{x}, \tilde{m})$ and $(y, p)$ solve the same differential systems. For Model (M1),

$$
\left\{\begin{array}{l}
\dot{m}_{1}+\dot{m}_{N}=\frac{m_{1}+m_{N}}{M} \sum_{j=2}^{N-1} m_{j}\left\langle\frac{\dot{x}_{1}+\dot{x}_{j}}{2}, u_{j 1}\right\rangle \\
\dot{m}_{i}=\frac{m_{i}}{M}\left(\left(m_{1}+m_{N}\right)\left\langle\frac{\dot{x}_{i}+\dot{x}_{1}}{2}, u_{1 i}\right\rangle+\sum_{j=2}^{N-1} m_{j}\left\langle\frac{\dot{x}_{i}+\dot{x}_{j}}{2}, u_{j i}\right\rangle\right), \quad i \in\{2, \cdots, N-1\} .
\end{array}\right.
$$

For Model (M2), since $x_{1}=x_{N}$, we have $p_{1}=p_{N}$, and so

$$
\left\{\begin{array}{l}
\dot{m}_{1}+\dot{m}_{N}=\frac{m_{1}+m_{N}}{M}\left(\frac{p_{1}}{\frac{1}{M}\left(\left(m_{1}+m_{N}\right) p_{1}+\sum_{j=1}^{N-1} m_{j} p_{j}\right)}-1\right) \\
\dot{m}_{i}=\frac{m_{i}}{M}\left(\frac{p_{i}}{\frac{1}{M}\left(\left(m_{1}+m_{N}\right) p_{1}+\sum_{j=1}^{N-1} m_{j} p_{j}\right)}-1\right), \quad i \in\{2, \ldots, N-1\} .
\end{array}\right.
$$

Then for either Model (M1) or Model (M2), $(\tilde{x}, \tilde{m})$ and $(y, q)$ follow the same dynamics, and since the initial conditions are the same, $(\tilde{x}, \tilde{m}) \equiv(y, q)$.

Hence, in Models (M1) and (M2), if for some $(i, j) \in\{1, \cdots, N\}^{2}, x_{i}^{0}=x_{j}^{0}$, we remove $x_{j}$ from the system and replace $m_{i}^{0}$ by $m_{i}^{0}+m_{j}^{0}$. The same cannot be done in the general case, when $\bar{\psi}_{i}$ depends explicitely on $m_{i}$.

Proposition 2. The specific models (M1), (M2), (M3) and (M4) satisfy Hypothesis 2. 
Proof. We compute $\sum_{i=1}^{N} \dot{m}_{i}$ for each model.

For Model 1 , the property $\sum_{i=1}^{N} \dot{m}_{i}=0$ follows easily from the antisymmetry of the summed coefficients.

For Model 2,

$$
\sum_{i=1}^{N} \dot{m}_{i}=\frac{\frac{1}{M} \sum_{i=1}^{N} m_{i} p_{i}}{\frac{1}{M} \sum j=1^{N} m_{j} p_{j}}-\frac{\sum_{i=1}^{N} m_{i}}{M}=1-1=0 .
$$

For Model 3,

$$
\sum_{i=1}^{N} \dot{m}_{i}=\sum_{i=1}^{N} m_{i}\left(\sum_{j \mid i \in I_{j}} \frac{m_{j}}{\left|I_{j}\right|_{m}}-1\right)=\sum_{j=1}^{N} \frac{m_{j}}{\left|I_{j}\right|_{m}} \sum_{i \in I_{j}} m_{i}-M=\sum_{j=1}^{N} \frac{m_{j}}{\left|I_{j}\right|_{m}}\left|I_{j}\right|_{m}-M=0 .
$$

Lastly, for Model 4,

$$
\sum_{i=1}^{N} \dot{m}_{i}=\sum_{i=1}^{N} m_{i}\left(\frac{1}{M} \sum_{j=1}^{N} m_{j} \frac{e_{j i}}{\frac{1}{M} \sum_{k=1}^{N} m_{k} e_{j k}}-1\right)=\sum_{j=1}^{N} m_{j} \frac{\frac{1}{M} \sum_{i=1}^{N} m_{i} e_{j i}}{\frac{1}{M} \sum_{k=1}^{N} m_{k} e_{j k}}-M=0 .
$$

We show that although the four specific models defined in Section 2.1 have generally discontinuous mass dynamics, some restrictions render $\psi$ continuous, and we can therefore consider their unique solutions in the classical sense.

Proposition 3. Let $(x, m)$ be a Filippov solution to system (M1), with $\psi_{i}(x, m)=$ $\frac{m_{i}}{M} \sum_{j=1}^{N} m_{j}\left\langle\frac{\dot{x}_{i}+\dot{x}_{j}}{2}, u_{j i}\right\rangle$. If for all $(i, j) \in\{1, \cdots, N\}^{2}, x_{i}^{0} \neq x_{j}^{0}$, then along the dynamics, $\psi_{i}$ is continuous with respect to $x$ and $m$ and $(x, m)$ is the unique solution of the Cauchy problem in the classical sense.

Proof. The only discontinuity of $\psi$ occurs when $x_{i}=x_{j}$ for some $(i, j) \in\{1, \cdots, N\}^{2}$. However, if initially $x_{i}^{0} \neq x_{j}^{0}$, for all $(i, j) \in\{1, \cdots, N\}^{2}$, we have $x_{i}(t) \neq x_{j}(t)$ for all $t \geq 0$. Hence along the dynamics, $\psi$ is locally Lipschitz with respect to $x$ and $m$ and each solution $(x, m)$ is continuously differentiable and satisfies (M1) for all $t \in \mathbb{R}$.

Proposition 4. Let $(x, m)$ be a Filippov solution to system (M2), with $\psi_{i}(x, m)=$ $\frac{m_{i}}{M}\left(p_{i}\left(\frac{1}{M} \sum_{j=1}^{M} m_{j} p_{j}\right)^{-1}-1\right)$, and suppose that $a(\cdot)$ is bounded away from zero. Then $\psi_{i}$ is only discontinuous at consensus, i.e. when $x_{i}=x_{j}$ for all $(i, j) \in\{1, \cdots, N\}^{2}$. If for all $(i, j) \in\{1, \cdots, N\}^{2}, x_{i}^{0} \neq x_{j}^{0}$, then along the dynamics, $\psi_{i}$ is continuous with respect to $x$ and $m$, and $(x, m)$ is the unique solution of the Cauchy problem in the classical sense.

Proof. The only possible point of discontinuity is at $(x, m)$ such that $\sum_{j=1}^{M} m_{j} p_{j}=0$. From Proposition 2, there exists at least one $i \in\{1, \cdots, N\}$ such that $m_{i} \neq 0$. Then since for all $j \in\{1, \cdots, N\}, m_{j} \geq 0$ and $p_{j} \geq 0$, we have $p_{i}=0$. Recall that the positional influence $p_{i}$ is defined by $p_{i}=\sum_{j=1}^{N} a\left(\left\|x_{i}-x_{j}\right\|\right)\left\|x_{i}-x_{j}\right\|$. Hence $p_{i}=0$ if and only if for all $j \in\{1, \cdots, N\},\left\|x_{i}-x_{j}\right\|=0$, that is at a consensus point. We now show 
that indeed $\psi_{i}$ is discontinuous around consensus. Consider two sequences $(x, m)$ and $(\tilde{x}, \tilde{m})$ converging to consensus while satisfying the following:

$$
\left\{\begin{array} { l } 
{ x _ { 1 } ( t ) \rightarrow _ { t \rightarrow \infty } 0 } \\
{ x _ { i } ( t ) = 0 \text { for all } i \geq 2 }
\end{array} \text { and } \left\{\begin{array}{l}
\tilde{x}_{2}(t)=-\tilde{x}_{1}(t) \rightarrow_{t \rightarrow \infty} 0 \\
\tilde{x}_{i}(t)=0 \text { for all } i \geq 3 .
\end{array}\right.\right.
$$

We compute for the first and the second sequence respectively:

$$
\left\{\begin{array} { l } 
{ p _ { 1 } = ( N - 1 ) a ( \| x _ { 1 } \| ) \| x _ { 1 } \| } \\
{ p _ { i } = a ( \| x _ { 1 } \| ) \| x _ { 1 } \| , i \geq 2 }
\end{array} \quad \left\{\begin{array}{l}
\tilde{p}_{1}=\tilde{p}_{2}=(N-2) a\left(\left\|\tilde{x}_{1}\right\|\right)\left\|\tilde{x}_{1}\right\|+2 a\left(2\left\|\tilde{x}_{1}\right\|\right)\left\|\tilde{x}_{1}\right\| \\
\tilde{p}_{i}=2 a\left(\left\|\tilde{x}_{1}\right\|\right)\left\|\tilde{x}_{1}\right\|, i \geq 3
\end{array}\right.\right.
$$

Then for the first sequence, for all $i \geq 2$,

$$
\frac{p_{i}}{\sum_{j=1}^{N} m_{j} p_{j}}=\frac{a\left(\left\|x_{1}\right\|\right)\left\|x_{1}\right\|}{\left(M-m_{1}\right) a\left(\left\|x_{1}\right\|\right)\left\|x_{1}\right\|+m_{1}(N-1) a\left(\left\|x_{1}\right\|\right)\left\|x_{1}\right\|}=\frac{1}{M+(N-2) m_{1}} .
$$

For the second sequence, for all $i \geq 3$,

$$
\frac{\tilde{p}_{i}}{\sum_{j=1}^{N} \tilde{m}_{j} \tilde{p}_{j}}=\frac{1}{\left(M-\tilde{m}_{12}\right)+\tilde{m}_{12}\left(\frac{N-2}{2}+\frac{a\left(2\left\|\tilde{x}_{1}\right\|\right)}{a\left(\left\|\tilde{x}_{1}\right\|\right)}\right)}=\frac{1}{M+\tilde{m}_{12}\left(\frac{N-4}{2}+\frac{a\left(2\left\|\tilde{\tilde{x}}_{1}\right\|\right)}{a\left(\left\|\tilde{x}_{1}\right\|\right)}\right)},
$$

where we denoted by $\tilde{m}_{12}$ the sum $\tilde{m}_{1}+\tilde{m}_{2}$. These quotients are in general different, hence $\psi_{i}(x, m)$ and $\psi_{i}(\tilde{x}, \tilde{m})$ converge to different limits as $x$ and $\tilde{x}$ converge to consensus. Therefore $\psi$ is discontinuous for all $(x, m)$ with $x$ at consensus. However, according to Proposition 1 , if initially $x_{i}^{0} \neq x_{j}^{0}$, for all $(i, j) \in\{1, \cdots, N\}^{2}$, we have $x_{i}(t) \neq x_{j}(t)$ for all $t \geq 0$. Hence along the dynamics, $\psi$ is locally Lipschitz with respect to $x$ and $m$ and each solution $(x, m)$ is continuously differentiable and satisfies (M1) in the classical sense. It is the unique solution to the Cauchy problem associated with (M2).

Proposition 5. Let $(x, m)$ be a Filippov solution to system (M4), with $\psi_{i}(x, m)=$ $m_{i}\left(\sum_{j=1}^{M} \frac{m_{j}}{M} e_{j i}\left(\frac{1}{M} \sum_{k=1}^{M} m_{k} e_{j k}\right)^{-1}-1\right)$, and suppose that $a(\cdot)$ is bounded away from zero. If for all $(i, j) \in\{1, \cdots, N\}^{2}, x_{i}^{0} \neq x_{j}^{0}$, then along the dynamics, $\psi_{i}$ is continuous with respect to $x$ and $m$, and $(x, m)$ is the unique solution of the Cauchy problem in the classical sense.

Proof. The only possible points of discontinuity are the points $(x, m)$ satisfying

$$
\sum_{k=1}^{M} m_{k}^{2} a\left(\left\|x_{j}-x_{k}\right\|\right)\left\|x_{j}-x_{k}\right\|=0
$$

for some $j \in\{1, \cdots, N\}$. Unlike for Model (M2), as seen in Proposition 4, this can occur for many configurations of the system, since the weights can be equal to zero. However, from Proposition 1, if for all $(i, j) \in\{1, \cdots, N\}^{2}, x_{i}^{0} \neq x_{j}^{0}$, then $x_{i}(t) \neq x_{j}(t)$ for all $t \geq 0$. Since for all time there exists $k \in\{1, \cdots, N\}$ such that $m_{k}(t) \neq 0$, for all $t \geq 0$ we have $\sum_{k=1}^{M} m_{k}(t)^{2} a\left(\left\|x_{j}(t)-x_{k}(t)\right\|\right)\left\|x_{j}(t)-x_{k}(t)\right\| \neq 0$. 
Remark 2. We have proven that under the assumption that $a$ is bounded below and that the system is initially not clustered, the functions driving the weight dynamics of models (M1), (M2) and (M4) are continuous along the trajectories of $x$ and $m$. We then consider solutions $(x, m)$ in the classical sense. This is not the case for model (M3) which is by nature discontinuous.

\subsection{General properties of the system}

Let

$$
x_{\max }^{0}:=\max _{i \in\{1, \cdots, N\}}\left\|x_{i}^{0}\right\| .
$$

We show that the system is not expanding in time in the following sense:

Proposition 6. Let $(x, m)$ be a solution to the general system (3)-(4)-(5). For all $t \in \mathbb{R}^{+}$,

$$
\max _{i \in\{1, \cdots, N\}}\left\|x_{i}(t)\right\| \leq x_{\max }^{0} .
$$

Proof. We show that $\max _{i \in\{1, \cdots, N\}}\left(\left\|x_{i}(t)\right\|\right)$ decreases with respect to time. For all $i \in\{1, \cdots, N\}$, if $\left\|x_{i}(t)\right\| \neq 0$,

$$
\begin{aligned}
\frac{d}{d t}\left\|x_{i}\right\| & =\frac{1}{2\left\|x_{i}\right\|} \frac{d}{d t}\left(\left\|x_{i}\right\|^{2}\right) \\
& =\frac{1}{\left\|x_{i}\right\|} \frac{1}{M} \sum_{j=1}^{N} m_{j} a\left(\left\|x_{i}-x_{j}\right\|\right)\left\langle x_{i}, x_{j}-x_{i}\right\rangle \\
& =\frac{1}{\left\|x_{i}\right\|} \frac{1}{M}\left(\sum_{j=1}^{N} m_{j} a\left(\left\|x_{i}-x_{j}\right\|\right)\left\langle x_{i}, x_{j}\right\rangle-\sum_{j=1}^{N} m_{j} a\left(\left\|x_{i}-x_{j}\right\|\right)\left\|x_{i}\right\|^{2}\right) .
\end{aligned}
$$

Let $i \in\{1, \cdots, N\}$ such that $\left\|x_{i}(t)\right\|=\max _{j \in\{1, \cdots, N\}}\left\|x_{j}(t)\right\|$. If $\left\|x_{i}(t)\right\|=0$, then (2) is trivial and $\left\|x_{i}\left(t^{\prime}\right)\right\|=0$ for all $t^{\prime} \geq t$. If $\left\|x_{i}(t)\right\| \neq 0$, then from (10), $\frac{d}{d t}\left\|x_{i}(t)\right\| \leq 0$, which proves (9).

An important consequence of the Lipschitz property of $s \mapsto a(\|s\|) s$ is that a solution to (2) cannot form clusters in finite time, as we show in the following proposition.

Proposition 7. Let $(x, m)$ be a solution to system (3)-(4)-(5), with initial condition $x(0)=x^{0}$. Then there exists $K \geq 0$ such that for all $(i, j) \in\{1, \cdots, N\}^{2}$,

$$
\left\|x_{i}(t)-x_{j}(t)\right\| \geq\left\|x_{i}(0)-x_{j}(0)\right\| e^{-K t} .
$$

Proof. From Proposition 6, for all $t \in \mathbb{R}^{+}$, for all $(i, j, k) \in\{1, \cdots, N\}^{3}, \|\left(x_{k}-x_{i}\right)-$ $\left(x_{k}-x_{j}\right)\|(t)=\| x_{i}-x_{j} \|(t) \leq 2 x_{\max }^{0}$. Then from Hypothesis 1 , there exists $K \geq 0$ 
(depending on $x_{\max }^{0}$ ) such that

$$
\begin{aligned}
\frac{d}{d t}\left\|x_{i}-x_{j}\right\|^{2} & =\frac{2}{M} \sum_{k=1}^{N} m_{k}\left\langle x_{i}-x_{j}, a\left(\left\|x_{k}-x_{i}\right\|\right)\left(x_{k}-x_{i}\right)-a\left(\left\|x_{k}-x_{j}\right\|\right)\left(x_{k}-x_{j}\right)\right\rangle \\
& \geq-\frac{2}{M} \sum_{k=1}^{N} m_{k}\left\|x_{i}-x_{j}\right\|\left\|a\left(\left\|x_{k}-x_{i}\right\|\right)\left(x_{k}-x_{i}\right)-a\left(\left\|x_{k}-x_{j}\right\|\right)\left(x_{k}-x_{j}\right)\right\| \\
& \geq-\frac{2}{M} \sum_{k=1}^{N} m_{k}\left\|x_{i}-x_{j}\right\| K\left\|\left(x_{k}-x_{i}\right)-\left(x_{k}-x_{j}\right)\right\|=-2 K\left\|x_{i}-x_{j}\right\|^{2}
\end{aligned}
$$

and we conclude with Gronwall's lemma.

We now examine the existence of solutions in the case of the four specific models (M1), (M2), (M3) and (M4). Let $\bar{x}:=\frac{1}{N} \sum_{i=1}^{N} x_{i}$ denote the average group opinion. We also define $\bar{X}:=\frac{1}{M} \sum_{i=1}^{N} m_{i} x_{i}$ the weighted average opinion. Recall that in the classical opinion dynamics model (1), the average opinion $\bar{x}$ is constant with respect to time.

Proposition 8. For the augmented model with varying weights (3)-(4)-(5), if the weights do not evolve in time (i.e. $\psi_{i} \equiv 0$ for all $i \in\{1, \cdots, N\}$ ), then the weighted average $\bar{X}=\frac{1}{M} \sum_{i=1}^{N} m_{i} x_{i}$ is constant with respect to time.

Proof. If $\dot{m}_{i}=0$ for all $i \in\{1, \cdots, N\}$,

$$
\frac{d}{d t} \bar{X}=\frac{1}{M} \sum_{i=1}^{N} m_{i} \dot{x}_{i}=\frac{1}{M} \sum_{i=1}^{N} \sum_{j=1}^{N} m_{i} m_{j} a\left(\left\|x_{i}-x_{j}\right\|\right)\left(x_{j}-x_{i}\right)=0
$$

by antisymmetry of the summed coefficients.

We now investigate the propensity of the system to form consensus. Let us suppose that for all $(i, j) \in\{1, \cdots, N\}^{2},\left\|x_{i}^{0}-x_{j}^{0}\right\|>0$. From Proposition 7, for all $(i, j) \in$ $\{1, \cdots, N\}^{2},\left\|x_{i}(t)-x_{j}(t)\right\| \geq\left\|x_{i}(0)-x_{j}(0)\right\| e^{-K t}$. From here onward we denote by

$$
d_{\min }(t):=\min _{(i, j) \in\{1, \cdots, N\}^{2}}\left\|x_{i}(0)-x_{j}(0)\right\| e^{-K t}
$$

the lower bound on the smallest distance between two agents at time $t$, i.e. for all $(i, j) \in\{1, \cdots, N\}^{2},\left\|x_{i}(t)-x_{j}(t)\right\| \geq d_{\min }(t)$. Furthermore, according to Proposition 6 , $\max _{i \in\{1, \cdots, N\}}\left\|x_{i}(t)\right\| \leq x_{\max }^{0}$. Then for all $t \in \mathbb{R}^{+}, 0<d_{\min }(t) \leq\left\|x_{i}(t)-x_{j}(t)\right\| \leq 2 x_{\max }^{0}$. Let us define the function $t \mapsto \alpha(t)$ as

$$
\alpha(t)=\sup _{z \in\left[d_{\min }(t), 2 x_{\max }^{0}\right]} a(z)
$$

For all $t \geq 0$, we then define a normalized interaction function $z \mapsto a_{t}(z)$ such that :

$$
a_{t}(z):=\frac{a(z)}{\alpha(t)}
$$


This normalized interaction function now satisfies the property $0 \leq a_{t}\left(\left\|x_{i}(t)-x_{j}(t)\right\|\right) \leq$ 1 for all $t \geq 0$ and all $(i, j) \in\{1, \cdots, N\}^{2}$. This will allow us to rewrite the system with a row-stochastic matrix $\left(A_{i j}\right)_{(i, j) \in\{1, \cdots, N\}^{2}}$ defined by:

$$
\left\{\begin{array}{l}
A_{i j}(t)=\frac{m_{j}(t)}{M} a_{t}\left(\left\|x_{i}(t)-x_{j}(t)\right\|\right) \quad \text { if } i \neq j \\
A_{i i}(t)=1-\sum_{j \neq i} \frac{m_{j}(t)}{M} a_{t}\left(\left\|x_{i}(t)-x_{j}(t)\right\|\right)
\end{array}\right.
$$

Then for all $t \geq 0$, for all $(i, j) \in\{1, \cdots, N\}^{2}, 0 \leq A_{i j}(t) \leq 1$. Moreover, we have constructed $A$ in such a way that for all $i \in\{1, \cdots, N\}, \sum_{j=1}^{N} A_{i j}(t)=1$, making it row-stochastic as claimed above.

Then denoting by $x(t)$ the opinion vector $\left(x_{i}\right)_{i \in\{1, \cdots, N\}}$, the opinion dynamics of system (2) can be rewritten in matrix form as:

$$
\dot{x}(t)=\alpha(t)(A(t) x(t)-x(t)) .
$$

Let $\Omega(t)$ denote the convex hull of $x(t)$, defined as follows.

Definition 2. Let $\left(x_{i}\right)_{i \in\{1, \cdots, N\}}$. Its convex hull $\Omega$ is defined by:

$$
\Omega=\left\{\sum_{i=1}^{N} \xi_{i} x_{i} \mid \forall i \in\{1, \cdots, N\}, 0 \leq \xi_{i} \leq 1 \text { and } \sum_{i=1}^{N} \xi_{i}=1\right\}
$$

We show that $\Omega(t)$ contracts in time with the following proposition.

Proposition 9. The convex hull $\Omega(t)$ of $x(t)$ is contracting in time in the sense that

$$
\Omega\left(t_{2}\right) \subseteq \Omega\left(t_{1}\right) \quad \text { for all } t_{2} \geq t_{1} \geq 0 .
$$

This implies that there exists a convex compact $\Omega_{\infty}$ such that

$$
\lim _{t \rightarrow+\infty} \Omega(t)=\Omega_{\infty}
$$

Proof. From Equation (15), we can rewrite the system as:

$$
\dot{x}_{i}(t)=\alpha(t)\left(\bar{x}_{i}(t)-x_{i}(t)\right), \quad i \in\{1, \cdots, N\},
$$

where $\bar{x}_{i}:=\sum_{j=1}^{N} A_{i j} x_{j}$ is a convex combination of the agents $x_{j}$. Then, by definition, $\bar{x}_{i}$ is inside the convex hull of $x$, and $\dot{x}_{i}$ is directed towards the convex hull, which ensures the property (16).

Theorem 1. Let $D: t \mapsto D(t):=\max _{(i, j) \in\{1, \cdots, N\}^{2}}\left\|x_{i}(t)-x_{j}(t)\right\|$ represent the diameter of the system. Then it satisfies the concentration estimate

$$
\frac{d}{d t} D(t) \leq-\alpha(t) \eta(t) D(t)
$$

with

$$
\alpha(t)=\sup \left\{a(z) \mid z \in\left[d_{\min }(t), 2 x_{\max }^{0}\right]\right\} \quad \text { and } \quad \eta(t):=\min _{(i, j) \in\{1, \cdots, N\}^{2}} \sum_{k=1}^{N} \min \left\{A_{i k}, A_{j k}\right\}
$$


for the row-stochastic matrix $A(t)$ defined in equation (14). and for the quantities $x_{\max }^{0}$ and $d_{\min }$ respectively defined in equations (8) and (11).

In particular, if $\lim _{t \rightarrow+\infty} \int_{0}^{t} \alpha(s) \eta(s) d s=+\infty$, then $\lim _{t \rightarrow+\infty} D(t)=0$. Furthermore, if $\int_{0}^{+\infty} \alpha(t) \exp \left(-\int_{0}^{t} \alpha(s) \eta(s) d s\right) d t<\infty$, then all the agents converge to an emerging consensus $x^{\infty}$ such that

$$
\left\|x_{i}(t)-x^{\infty}\right\| \leq \exp \left(-\int_{0}^{t} \alpha(s) \eta(s) d s\right) D(0) .
$$

Proof. After writing the system in matrix form (15), where $A$ is row-stochastic, the proof is an easy adaptation of the proof of Theorem 2.2 of [23]. Let us first show that

$$
\max _{(i, j) \in\{1, \cdots, N\}^{2}}\left\|(A(t) x(t))_{i}-(A(t) x(t))_{j}\right\| \leq(1-\eta(t)) D(t) .
$$

Let $(i, j) \in\{1, \cdots, N\}^{2}$ and let $\eta_{k}^{i j}:=\min \left\{A_{i j}, A_{j k}\right\}$. We omit the time $t$ for convenience of notation and compute:

$$
\begin{aligned}
\left\|(A x)_{i}-(A x)_{j}\right\| & =\left\|\sum_{k=1}^{N} A_{i j} x_{k}-\sum_{k=1}^{N} A_{j k} x_{k}\right\|=\left\|\sum_{k=1}^{N}\left(A_{i j}-\eta_{k}^{i j}\right) x_{k}-\sum_{k=1}^{N}\left(A_{j k}-\eta_{k}^{i j}\right) x_{k}\right\| \\
& \leq \sum_{k=1}^{N}\left(A_{i j}-\eta_{k}^{i j}\right) \max _{k \in\{1, \cdots, N\}}\left\|x_{k}\right\|-\sum_{k=1}^{N}\left(A_{j k}-\eta_{k}^{i j}\right) \min _{k \in\{1, \cdots, N\}}\left\|x_{k}\right\| \\
& \leq\left(1-\sum_{k=1}^{N} \eta_{k}^{i j}\right)\left(\max _{k \in\{1, \cdots, N\}}\left\|x_{k}\right\|-\min _{k \in\{1, \cdots, N\}}\left\|x_{k}\right\|\right) \\
& \leq(1-\eta) \max _{\left.(i, j) \in\{1, \cdots, N\}^{2}\right)}\left\|x_{i}-x_{j}\right\|=(1-\eta) D .
\end{aligned}
$$

Let us now compute from (15):

$$
\begin{aligned}
\frac{d}{d t}\left\|x_{i}-x_{j}\right\|^{2} & =2\left\langle x_{i}-x_{j}, \alpha\left(\sum_{k=1}^{N} A_{i k} x_{k}-x_{i}\right)-\alpha\left(\sum_{k=1}^{N} A_{j k} x_{k}-x_{j}\right)\right\rangle \\
& \leq 2 \alpha\left(-\left\|x_{i}-x_{j}\right\|^{2}+\left\|(A x)_{i}-(A x)_{j}\right\|\left\|x_{i}-x_{j}\right\|\right) \\
& \leq 2 \alpha\left(-\left\|x_{i}-x_{j}\right\|^{2}+(1-\eta) D\left\|x_{i}-x_{j}\right\|\right)
\end{aligned}
$$

Let $(i, j) \in\{1, \cdots, N\}^{2}$ such that $\left\|x_{i}-x_{j}\right\|=D$. Then

$$
\frac{d}{d t} D(t)^{2} \leq 2 \alpha(t)\left(-D(t)^{2}+(1-\eta(t)) D(t)^{2}\right)=-2 \alpha(t) \eta(t) D(t)^{2},
$$

which implies (18). It follows that $D(t)=D(0) \exp \left(-\int_{0}^{t} \alpha(s) \eta(s) d s\right)$, so

$$
\lim _{t \rightarrow+\infty} \int_{0}^{t} \alpha(s) \eta(s) d s=+\infty \Rightarrow \lim _{t \rightarrow+\infty} D(t)=0 .
$$


Then the diameter of the system converges to zero. We further show that each agent converges to consensus. Let $i \in\{1, \cdots, N\}$, and let $0 \leq t_{1}<t_{2}$.

$$
\begin{aligned}
\left\|x_{i}\left(t_{2}\right)-x_{i}\left(t_{1}\right)\right\| & =\left\|\int_{t_{1}}^{t_{2}} \dot{x}_{i} d t\right\|=\left\|\int_{t_{1}}^{t_{2}} \alpha(t)\left((A x)_{i}(t)-x_{i}(t)\right) d t\right\| \\
& \leq \int_{t_{1}}^{t_{2}} \alpha(t) \max _{(i, j) \in\{1, \cdots, N\}^{2}}\left\|x_{i}(t)-x_{j}(t)\right\| d t=\int_{t_{1}}^{t_{2}} \alpha(t) D(t) \\
& \leq D(0) \int_{t_{1}}^{t_{2}} \alpha(t) \exp \left(-\int_{0}^{t} \alpha(s) \eta(s) d s\right) d t
\end{aligned}
$$

Hence,

$\lim _{\tau \rightarrow+\infty} \int_{0}^{\tau} \alpha(t) \exp \left(-\int_{0}^{t} \alpha(s) \eta(s) d s\right) d t<+\infty \Rightarrow \lim _{\left|t_{1}-t_{2}\right| \rightarrow 0}\left\|x_{i}\left(t_{2}\right)-x_{i}\left(t_{1}\right)\right\|=0$,

which implies the convergence of $x_{i}$ to a consensus opinion $x^{\infty}$ satisfying (19).

\subsection{Consensus in the case of a positive interaction function}

We now show that if the interaction function $a$ is bounded below on the initial diameter of the system, then the system converges to consensus.

Proposition 10. Let $(x, m)$ be a solution to (3)-(4)-(5) and let $t \mapsto D(t)$ be the diameter of the system as defined in Theorem 1. If $\inf \{a(z) \mid z \leq D(0)\}:=a_{\min }>0$ then the system (2) converges to consensus, with the rate

$$
D(t) \leq D(0) e^{-a_{\min } t}
$$

Proof. Due to the assumption on $a$, at $t=0$, since for all $(i, j) \in\{1, \cdots, N\}^{2}, \| x_{i}^{0}-$ $x_{j}^{0} \| \leq D(0)$, we have $a\left(\left\|x_{i}^{0}-x_{j}^{0}\right\|\right) \geq a_{\min }$. According to Theorem 1 , for all $t \in \mathbb{R}^{+}$, $D(t) \leq D(0)$, so for all $t \in \mathbb{R}^{+}$, for all $(i, j) \in\{1, \cdots, N\}^{2}, a\left(\left\|x_{i}(t)-x_{j}(t)\right\|\right) \geq a_{\min }$. Consider $(i, j) \in\{1, \cdots, N\}^{2}$ such that $D(t)=\left\|x_{i}(t)-x_{j}(t)\right\|=\max _{(k, l) \in\{1, \cdots, N\}^{2}} \| x_{k}(t)-$ $x_{l}(t) \|$. We compute $\frac{d}{d t} D(t)^{2}=\frac{d}{d t}\left\|x_{i}(t)-x_{j}(t)\right\|^{2}$ :

$$
\begin{aligned}
\frac{d}{d t}\left(\left\|x_{i}-x_{j}\right\|^{2}\right)= & \frac{2}{M} \sum_{k=1}^{N} m_{k} a\left(\left\|x_{i}-x_{k}\right\|\right)\left\langle x_{k}-x_{i}, x_{i}-x_{j}\right\rangle \\
& -\frac{2}{M} \sum_{k=1}^{N} m_{k} a\left(\left\|x_{j}-x_{k}\right\|\right)\left\langle x_{k}-x_{j}, x_{i}-x_{j}\right\rangle .
\end{aligned}
$$

Because $\left\|x_{i}(t)-x_{j}(t)\right\|=D(t)$, for all $(k, l) \in\{1, \cdots, N\}^{2},\left\langle x_{k}-x_{i}, x_{i}-x_{j}\right\rangle \leq 0$ and symmetrically, $\left\langle x_{k}-x_{j}, x_{i}-x_{j}\right\rangle \geq 0$. Consequently,

$$
\begin{aligned}
\frac{d}{d t}\left(\left\|x_{i}-x_{j}\right\|^{2}\right) & \leq \frac{2}{M} \sum_{k=1}^{N} m_{k} a_{\min }\left\langle x_{k}-x_{i}, x_{i}-x_{j}\right\rangle-\frac{2}{M} \sum_{k=1}^{N} m_{k} a_{\min }\left\langle x_{k}-x_{j}, x_{i}-x_{j}\right\rangle \\
& \leq-\frac{2}{M} a_{\min } \sum_{k=1}^{N} m_{k}\left\|x_{i}-x_{j}\right\|^{2} \leq-2 a_{\min }\left\|x_{i}-x_{j}\right\|^{2} .
\end{aligned}
$$

Then $D(t) \leq D(0) e^{-a_{\min } t}$, which implies that the system converges to consensus. 
Remark 3. The condition on the boundedness away from zero of the interaction function does not prevent the effective interactions $m_{k} a\left(\left\|x_{i}-x_{k}\right\|\right)$ from being zero, as the individual weights $m_{k}$ can tend to 0 . However, due to the positivity of the total mass of the system $M>0$, the directed graph of interactions remains weakly connected and consensus is still achieved.

\subsection{Clustering in the case of an interaction function with bounded support}

In the previous section, we saw that a positive interaction function leads to consensus of the system. We now explore the behavior of the system in the case of an interaction function with bounded support, i.e. we suppose the following:

Hypothesis 3. Suppose that there exists $R \geq 0$ such that $a$ satisfies

$$
\left\{\begin{array}{l}
a(r)>0 \text { for all } r \in(0, R) \\
a(r)=0 \text { for all } r \geq R
\end{array}\right.
$$

In the case of the classical Hegselmann-Krause model (1), this was shown to lead to clustering equilibria [18], i.e. states of the system in which for all $(i, j) \in\{1, \cdots, N\}^{2}$, either $\left\|x_{i}-x_{j}\right\|=0$ or $\left\|x_{i}-x_{j}\right\| \geq R$, where $R$ denotes the size of the support of the interaction function. In this section, we prove that in the case of non-evolving positive weights, we obtain the same result. The case of non-evolving positive weights is covered by the framework of type-symmetric and cut-balanced systems, and convergence was shown by Hendrickx and Tsitsiklis in [17]. Cut-balanced systems model situations in which if a group of agents influences another group, then the first group is also influenced by the second one by at least a proportional amount. In this paper, we consider the opposite situation, by allowing a subgroup of agents to become the only ones with influence. Our system is cut-balanced only when the weights are bounded away from zero. We saw in Section 2.4 that as long as the total mass $M$ is positive, if the interaction function is positive, the system converges to consensus, even if some weights vanish. We now examine the case of an interaction function with bounded support. We show that we can adapt the work of Jabin and Motsch [18] to prove the clustering of the system, with the strong condition that the weights are positive and do not evolve. We also show that when the masses evolve and are allowed to vanish, we cannot expect the results to hold.

Let the energy of the system be defined by:

$$
E(x)=\frac{1}{M^{2}} \sum_{i=1}^{N} \sum_{j=1}^{N} m_{i} m_{j} \Phi\left(\left\|x_{i}-x_{j}\right\|^{2}\right), \quad \Phi: r \mapsto \int_{0}^{r} a(\sqrt{s}) d s .
$$

Proposition 11. Consider the simplified system (3)-(4)-(5) with constant masses, i.e. $\Psi_{i} \equiv 0$ for all $i \in\{1, \cdots, N\}$. Then the energy decreases in time with:

$$
\frac{d E}{d t}=-\frac{4}{M} \sum_{i=1}^{N} m_{i}\left\|\dot{x}_{i}\right\|^{2}
$$


and

$$
\frac{d E}{d t} \leq-\frac{1}{M^{3}} \frac{\left(\sum_{i=1}^{N} \sum_{j=1}^{N} m_{i} m_{j} a\left(\left\|x_{i}-x_{j}\right\|\right)\left\|x_{i}-x_{j}\right\|^{2}\right)^{2}}{\sum_{i=1}^{N} m_{i}\left\|x_{i}\right\|^{2}} .
$$

Furthermore, the following inequality follows:

$$
\int_{0}^{+\infty} m_{i}^{2} m_{j}^{2} a^{2}\left(\left\|x_{i}(t)-x_{j}(t)\right\|\right)\left\|x_{i}(t)-x_{j}(t)\right\|^{4} d t \leq C_{M}
$$

where $C_{M}$ is a constant depending only on the total mass $M$.

Proof. For the equality (23), we compute the time derivative of the energy functional when the masses are constant in time:

$$
\begin{aligned}
\frac{d E}{d t} & =\frac{1}{M^{2}} \sum_{i=1}^{N} \sum_{j=1}^{N} m_{i} m_{j} a\left(\left\|x_{i}-x_{j}\right\|\right) 2\left\langle x_{i}-x_{j}, \dot{x}_{i}-\dot{x}_{j}\right\rangle \\
& =\frac{4}{M^{2}} \sum_{i=1}^{N} \sum_{j=1}^{N} m_{i} m_{j} a\left(\left\|x_{i}-x_{j}\right\|\right)\left\langle x_{i}-x_{j}, \dot{x}_{i}\right\rangle=-\frac{4}{M} \sum_{i=1}^{N} m_{i}\left\|\dot{x}_{i}\right\|^{2} .
\end{aligned}
$$

Furthermore, from the Cauchy-Schwarz inequality,

$$
\left(\sum_{i=1}^{N} m_{i}\left\langle\dot{x}_{i}, x_{i}\right\rangle\right)^{2} \leq\left(\sum_{i=1}^{N} m_{i}\left\|\dot{x}_{i}\right\|^{2}\right)\left(\sum_{i=1}^{N} m_{i}\left\|x_{i}\right\|^{2}\right)=-\frac{M}{4} \frac{d E}{d t}\left(\sum_{i=1}^{N} m_{i}\left\|x_{i}\right\|^{2}\right),
$$

from which we get

$$
\left(\sum_{i=1}^{N} \frac{1}{M} \sum_{j=1}^{N} m_{i} m_{j} a\left(\left\|x_{i}-x_{j}\right\|\right)\left\langle x_{j}-x_{i}, x_{i}\right\rangle\right)^{2} \leq-\frac{M}{4} \frac{d E}{d t}\left(\sum_{i=1}^{N} m_{i}\left\|x_{i}\right\|^{2}\right),
$$

so

$$
\left(\frac{1}{2 M} \sum_{i=1}^{N} \sum_{j=1}^{N} m_{i} m_{j} a\left(\left\|x_{i}-x_{j}\right\|\right)\left\|x_{i}-x_{j}\right\|^{2}\right)^{2} \leq \frac{M}{4} \frac{d E}{d t}\left(\sum_{i=1}^{N} m_{i}\left\|x_{i}\right\|^{2}\right),
$$

and this gives the desired inequality (24). Lastly, from Proposition 6, the opinion vector $x$ is bounded at all time, so there exists $C_{M}$ such that

$$
\int_{0}^{+\infty} \sum_{i=1}^{N} \sum_{j=1}^{N} m_{i}^{2} m_{j}^{2} a^{2}\left(\left\|x_{i}(t)-x_{j}(t)\right\|\right)\left\|x_{i}(t)-x_{j}(t)\right\|^{4} d t \leq C_{M},
$$

which implies the inequality (25).

Proposition 11 implies that the stationary states $S$ of the system are the states satisfying $\frac{d E}{d t}=0$, i.e. if $a$ satisfies Hypothesis 3,

$$
S=\left\{x \in\left(\mathbb{R}^{d}\right)^{N} \mid \forall(k, l) \in\{1, \cdots, N\}^{2}, m_{k} m_{l}=0 \text { or }\left\|x_{k}-x_{l}\right\|=0 \text { or }\left\|x_{k}-x_{l}\right\| \geq R\right\} .
$$


We now prove that if the masses are all strictly positive, the system converges to an equilibrium satisfying:

$$
\forall(k, l) \in\{1, \cdots, N\}^{2},\left\|x_{k}-x_{l}\right\|=0 \text { or }\left\|x_{k}-x_{l}\right\| \geq R .
$$

Proposition 12. Suppose that a(.) satisfies Hypothesis 3. Suppose that $\psi_{i} \equiv 0$ and $m_{i}>0$ for all $i \in\{1, \cdots, N\}$. Then for all $\varepsilon>0$, there exists $T_{\varepsilon}$ such that for all $(i, j) \in\{1, \cdots, N\}^{2}$, for all $t \geq T_{\mathcal{\varepsilon}},\left\|x_{i}(t)-x_{j}(t)\right\| \in[0, \varepsilon] \cup[R-\varepsilon,+\infty)$.

Proof. By contradiction, suppose that there exists $(i, j) \in\{1, \cdots, N\}$ and $\left\{t_{n}\right\}_{n \in \mathbb{N}}$ such that for all $n \in \mathbb{N},\left\|x_{i}\left(t_{n}\right)-x_{j}\left(t_{n}\right)\right\| \in(\varepsilon, R-\varepsilon)$. From the hypotheses on $a(\cdot)$, there exists $\delta>0$ such that for all $r \in(\varepsilon, R-\varepsilon), a(r) \geq \delta$. Furthermore, since from Theorem 1 , for all $t \geq 0,\left\|x_{i}(t)-x_{j}(t)\right\| \leq D(0)$, from Hypothesis 1 there exists $C>0$ such that for all $t \geq 0, a\left(\left\|x_{i}-x_{j}\right\|\right)\left\|x_{i}-x_{j}\right\| \leq C$. Then

$$
\left\|\dot{x}_{i}\right\| \leq \frac{1}{M} \sum_{j=1}^{N} m_{j} a\left(\left\|x_{i}-x_{j}\right\|\right)\left\|x_{i}-x_{j}\right\| \leq \frac{1}{M} \sum_{j=1}^{N} m_{j} C \leq C,
$$

so $x$ is uniformly continuous in time. This implies that there exists $\tau>0$ such that $a\left(\left\|x_{i}(t)-x_{j}(t)\right\|\right) \geq \frac{\delta}{2}$ and $\left\|x_{i}(t)-x_{j}(t)\right\| \geq \frac{\varepsilon}{2}$ for all $r \in\left[t_{n}, t_{n}+\tau\right]$, for all $n \in \mathbb{N}$. Then

$$
\begin{aligned}
& \lim _{T \rightarrow+\infty} \int_{0}^{T} m_{i}^{2} m_{j}^{2} a\left(\left\|x_{i}(t)-x_{j}(t)\right\|\right)^{2}\left\|x_{i}(t)-x_{j}(t)\right\|^{4} d t \\
\geq & \lim _{k \rightarrow+\infty} \sum_{n=1}^{k} \int_{t_{n}}^{t_{n}+\tau} m_{i}^{2} m_{j}^{2} a\left(\left\|x_{i}(t)-x_{j}(t)\right\|\right)^{2}\left\|x_{i}(t)-x_{j}(t)\right\|^{4} d t \\
\geq & \lim _{k \rightarrow+\infty} \sum_{n=1}^{k} \tau \frac{\delta^{2}}{4} \frac{\varepsilon^{4}}{16} m_{\min }^{4}=+\infty
\end{aligned}
$$

where $m_{\min }:=\min _{i \in\{1, \cdots, N\}}\left\{m_{i}\right\}$. This contradicts the inequality (25).

The next corollary follows automatically from Proposition 12:

Corollary 2. If a satisfies (21), and $\psi_{i} \equiv 0, m_{i}>0$ for all $i \in\{1, \cdots, N\}$, the system forms clusters separated by a distance greater than $R$, i.e. for any $(i, j) \in\{1, \cdots, N\}^{2}$,

$$
\lim _{t \rightarrow+\infty}\left\|x_{i}(t)-x_{j}(t)\right\|=0 \quad \text { or } \quad \lim _{t \rightarrow+\infty}\left\|x_{i}(t)-x_{j}(t)\right\| \geq R .
$$

Corollary 1 implies that the solution to (2) with constant positive weights forms clusters, but it is not enough in order to prove its convergence. In dimension 1, the convergence of the system is a direct consequence of the preserved order stated in Proposition 1, of the clustering property stated in Corollary 2 and of the convergence of the convex hull $\Omega$ stated in Proposition 9. The proof is identical to that of Theorem 4 in [18], hence we do not provide the details. Instead, we focus on the multidimensional case, which is more complicated due to the fact that lost connectivity between two agents can be recovered at a later time. To prove convergence of the solution to System (2) with constant positive weights, we follow the method introduced in [18]. We start by refining the result of Proposition 11: 
Proposition 13. Let $(x, m)$ be a solution to the simplified system (3)-(4)-(5) with constant masses, i.e. $\Psi_{i} \equiv 0$ for all $i \in\{1, \cdots, N\}$. For all $(i, j) \in\{1, \cdots, N\}^{2}$, for all $T \geq 0$,

$$
\int_{0}^{T} m_{i} m_{j} a\left(\left\|x_{i}(t)-x_{j}(t)\right\|\right)\left\|x_{i}(t)-x_{j}(t)\right\|^{2} d t \leq \frac{M^{2}}{4}\left(x_{\max }^{0}\right)^{2} .
$$

Proof. We start by writing:

$$
\int_{0}^{T} \sum_{i=1}^{N} m_{i}\left\langle x_{i}(t), \dot{x}_{i}(t)\right\rangle d t=\int_{0}^{T} \sum_{i=1}^{N} \sum_{j=1}^{N} \frac{m_{i} m_{j}}{M} a\left(\left\|x_{i}(t)-x_{j}(t)\right\|\right)\left\langle x_{i}(t), x_{j}(t)-x_{j}(t)\right\rangle d t .
$$

Then we have:

$$
\int_{0}^{T} \sum_{i=1}^{N} \sum_{j=1}^{N} \frac{m_{i} m_{j}}{M} a\left(\left\|x_{i}(t)-x_{j}(t)\right\|\right)\left\|x_{i}(t), x_{j}(t)\right\|^{2} d t=-\frac{1}{4} \int_{0}^{T} \sum_{i=1}^{N} m_{i} \frac{d}{d t}\left\|x_{i}(t)\right\|^{2} d t .
$$

The right-hand side can be written as:

$$
-\frac{1}{4} \int_{0}^{T} \sum_{i=1}^{N} m_{i} \frac{d}{d t}\left\|x_{i}(t)\right\|^{2} d t=-\frac{1}{4}\left[\sum_{i=1}^{N} m_{i}\left\|x_{i}(t)\right\|^{2}\right]_{0}^{T}=\frac{M}{4}\left(\left(x_{\max }^{0}\right)^{2}-\left(x_{\max }^{T}\right)^{2}\right),
$$

where we used the boundedness of $\left\|x_{i}\right\|$ proven in Proposition 6. Inequality (27) follows from the non-negativity of each term.

We are now equipped to prove the convergence theorem. From Corollary 2, we know that the agents form clusters separated by distances at least equal to $R$, the support of the interaction function.

Theorem 2. Let $(x, m)$ be a solution to (3)-(4)-(5). Suppose that a $(\cdot)$ satisfies Hypothesis 3 , and $\psi_{i} \equiv 0, m_{i}>0$ for all $i \in\{1, \cdots, N\}$. Let $N_{C} \leq N$ denote the number of clusters formed by the system. We denote by $\mathscr{C}_{k}$ the set of agents forming the $k-t h$ cluster, by $M_{k}:=\sum_{i \in \mathscr{C}_{k}} m_{i}$ the mass of $\mathscr{C}_{k}$, and by $y_{k}:=\frac{1}{M_{k}} \sum_{i \in \mathscr{C}_{k}} m_{i} x_{i}$ its barycenter. Then there exists a constant $C$ such that for each $k \in\left\{1, \ldots, N_{C}\right\}$, for all $T \geq 0$, the barycenter of $\mathscr{C}_{k}$ satisfies:

$$
\int_{0}^{T}\left\|\dot{y}_{k}(t)\right\| d t \leq C .
$$

Hence the system (2) with constant weights converges as $t \rightarrow+\infty$.

Proof. Let $k \in\left\{1, \ldots, N_{C}\right\}$. We compute the velocity of the center of mass $y_{k}$ of the cluster $\mathscr{C}_{k}$ :

$$
\begin{aligned}
\dot{y}_{k} & =\frac{1}{M_{k}} \sum_{i \in \mathscr{C}_{k}} m_{i} \dot{x}_{i} \\
& =\frac{1}{M_{k}} \sum_{i \in \mathscr{C}_{k}}\left[\sum_{j \in \mathscr{C}_{k}} \frac{m_{i} m_{j}}{M} a\left(\left\|x_{i}-x_{j}\right\|\right)\left(x_{j}-x_{i}\right)+\sum_{j \notin \mathscr{C}_{k}} \frac{m_{i} m_{j}}{M} a\left(\left\|x_{i}-x_{j}\right\|\right)\left(x_{j}-x_{i}\right)\right] .
\end{aligned}
$$


The first term vanishes by antisymmetry of the summed coefficients. For the second one, we sum over $i \in \mathscr{C}_{k}$ and $j \notin \mathscr{C}_{k}$. Then as a consequence of Corollary 2, there exists $T^{\prime} \geq 0$ such that for all $t \geq T^{\prime},\left\|x_{i}-x_{j}\right\| \geq \frac{R}{2}$. Hence for all $t \geq T^{\prime}, \frac{1}{R}\left\|x_{i}-x_{j}\right\| \leq$ $\frac{2}{R^{2}}\left\|x_{i}-x_{j}\right\|^{2}$. If $T^{\prime} \leq T$, the integral between $T^{\prime}$ and $T$ can be bounded as follows:

$$
\begin{aligned}
& \int_{T^{\prime}}^{T} \sum_{i \in \mathscr{C}_{k}} \sum_{j \notin \mathscr{C}_{k}} \frac{m_{i} m_{j}}{M M_{k}} a\left(\left\|x_{i}(t)-x_{j}(t)\right\|\right)\left\|x_{j}(t)-x_{i}(t)\right\| d t \\
\leq & \int_{T^{\prime}}^{T} \sum_{i \in \mathscr{C}_{k}} \sum_{j \notin \mathscr{C}_{k}} \frac{m_{i} m_{j}}{M M_{k}} a\left(\left\|x_{i}(t)-x_{j}(t)\right\|\right) \frac{2}{R}\left\|x_{j}(t)-x_{i}(t)\right\|^{2} d t \leq \frac{M^{2}}{2 R}\left(x_{\max }^{0}\right)^{2}
\end{aligned}
$$

where we used the inequality (27). Furthermore, as a consequence of Hypothesis 1 and of Theorem 1 , there exists $C^{\prime}>0$ such that for all $t \geq 0, a\left(\left\|x_{i}(t)-x_{j}(t)\right\|\right) \| x_{j}(t)-$ $x_{i}(t) \| \leq C^{\prime}$.

$$
\int_{0}^{T^{\prime}} \sum_{i \in \mathscr{C}_{k}} \sum_{j \notin \mathscr{C}_{k}} \frac{m_{i} m_{j}}{M M_{k}} a\left(\left\|x_{i}(t)-x_{j}(t)\right\|\right)\left\|x_{j}(t)-x_{i}(t)\right\| d t \leq T^{\prime} C^{\prime}
$$

Then (28) follows with $C:=\frac{M^{2}}{2 R}\left(x_{\max }^{0}\right)^{2}+T^{\prime} C^{\prime}$ if $T \geq T^{\prime}$, and $C:=T^{\prime} C^{\prime}$ if $T^{\prime} \geq$ $T$. We deduce that the barycenters $y_{k}$ of the clusters converge as $t \rightarrow+\infty$, and from Corollary 2, this implies that $x_{i}$ converges for each $i \in\{1, \cdots, N\}$.

Remark 4. The proof of Theorem 1 follows closely the proof of Proposition 3.2 in [18]. The main difference is that we prove the convergence of the barycenters of each cluster, instead of their centers. This allows us to recover a symmetry property that greatly simplifies the computation of $\dot{y}_{k}$.

Hence the system with non-evolving positive masses behaves in the same way as the non-weighted dynamics (see [18]). However, as soon as one mass vanishes, we may observe clusters separated by any distance. The following simple 1-dimensional example shows such a case of equilibrium at a distance smaller than $R$.

Example 1. Let $(x, m)$ be a solution to (3)-(4)-(5) with $d=1$ and $N=3$. Suppose that $a(\cdot)$ satisfies Hypothesis 3 , and $\left|x_{1}-x_{2}\right|=R$, with $m_{3}=0$. Then $\dot{x}_{1}=\dot{x}_{2}=0$, and the system is at equilibrium if $\dot{m}_{1}=\dot{m}_{2}=\dot{m}_{3}=\dot{x}_{3}=0$, i.e. if $\psi_{1}(x, m)=\psi_{2}(x, m)=$ $\psi_{3}(x, m)=0$ and

$$
m_{1} a\left(\left|x_{1}-x_{3}\right|\right)\left(x_{1}-x_{3}\right)+m_{2} a\left(\left|x_{2}-x_{3}\right|\right)\left(x_{2}-x_{3}\right)=0
$$

Simplifying even further, if we consider Model (M1) and if we suppose $a(r)=1$ for all $r \in[0, R)$, the system is at equilibrium if

$$
\frac{\left|x_{2}-x_{3}\right|}{\left|x_{1}-x_{3}\right|}=\frac{m_{1}}{m_{2}}
$$

Simulations in the case of time-evolving masses show other cases of equilibria with clusters separated by distances smaller than $R$. 

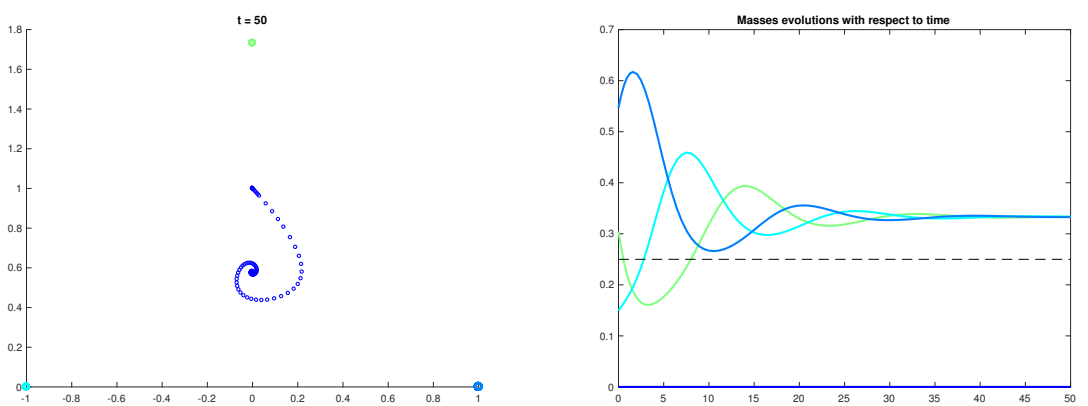

Figure 1: Evolution of the system corresponding to Example 2. Left: Evolution of the positions $\left\{x_{i}\right\}_{i \in\{1, \ldots, 4\}}$. Right: Evolution of the masses $\left\{m_{i}\right\}_{i \in\{1, \ldots, 4\}}$. The dashed line shows the constant average mass of the system.

Example 2. Let $d=2$ and $N=4$. Suppose that $a(r)=1$ for $r \in\left[0, \frac{3}{2}\right)$ and 0 otherwise. We place three agents at the vertices of an equilateral triangle of side equal to 2 , with $x_{1}=(0, \sqrt{3}), x_{2}=(-1,0)$ and $x_{3}=(1,0)$. We suppose that $m_{4}=0$ and we simulate the evolution of the system with the mass dynamics given by Model (M2). The positions of the the first three agents stay invariant in time with $\dot{x}_{1}=\dot{x}_{2}=\dot{x}_{3}=0$, and the mass of the fourth agent remains equal to 0 . Then the only variable quantities are $x_{4}$, and the three masses $m_{1}, m_{2}$ and $m_{3}$. Simulations show that if $\left\|x_{4}-x_{i}\right\|<R$ is satisfied for all $i \in\{1, \ldots, 3\}$, the system tends to an equilibrium given by $m_{1}=m_{2}=m_{3}=\frac{1}{3}$ and $x_{4}=\left(\frac{1}{\sqrt{3}}, 0\right)$ being the center of the circumscribing circle of $\left(x_{1}, x_{2}, x_{3}\right)$, see Figure 1. Furthermore, although the system converges to equilibrium, we observe oscillations both in the mass evolution and in the spiral trajectory of $x_{4}$.

\section{Long-term behavior and equilibria for heterophilious dynamics}

We now focus on the specific case of a constant interaction function, for example $a \equiv 1$. This models the case of heterophilious dynamics, as the influence of one agent over another grows with the distance separating them [23]. We use this case of simple interaction function to illustrate some fundamental differences between the four models (M1), (M2), (M3) and (M4), more specifically in the long-term behavior of the weights and in the existence of equilibria.

Proposition 14. Suppose that the interaction function is constant, $a \equiv 1$. Then the solution to system (3)-(4)-(5) converges exponentially to consensus, and we have:

$$
\frac{d}{d t} D(t) \leq D(0) e^{-t}
$$

Furthermore, the system converges to the consensus opinion $x^{\infty}$ and

$$
\forall i \in\{1, \cdots, N\}, \quad\left\|x_{i}(t)-x^{\infty}\right\| \leq e^{-t} D(0) .
$$


Lastly,

$$
\forall(i, j) \in\{1, \cdots, N\}^{2}, \quad x_{i}(t)-x_{j}(t)=e^{-t}\left(x_{i}^{0}-x_{j}^{0}\right) .
$$

Proof. With $a \equiv 1$, we have $\alpha \equiv 1$ and the opinion dynamics can be written in matrix form (15) with the row-stochastic matrix $A$ defined as:

$$
\left\{\begin{array}{l}
A_{i j}(t)=\frac{m_{j}(t)}{M} \quad \text { if } i \neq j \\
A_{i i}(t)=1-\sum_{j \neq i} \frac{m_{j}(t)}{M}
\end{array}\right.
$$

We apply Theorem 1 and calculate:

$$
\begin{aligned}
& \sum_{k=1}^{N} \min \left\{A_{i k}, A_{j k}\right\} \\
= & \sum_{k \neq i, j}^{N} \min \left\{\frac{m_{k}(t)}{M}, \frac{m_{k}(t)}{M}\right\}+\min \left\{1-\sum_{k \neq i} \frac{m_{k}(t)}{M}, \frac{m_{i}(t)}{M}\right\}+\min \left\{1-\sum_{k \neq j} \frac{m_{k}(t)}{M}, \frac{m_{j}(t)}{M}\right\} \\
= & \sum_{k \neq i, j}^{N} \frac{m_{k}(t)}{M}+\frac{m_{i}(t)}{M}+\frac{m_{j}(t)}{M}=1 .
\end{aligned}
$$

Then $\eta(t)=\min _{(i, j) \in\{1, \cdots, N\}^{2}} 1=1$, and we get (29) from (18). Furthermore,

$$
\int_{0}^{+\infty} \exp \left(-\int_{0}^{t} \eta(s) d s\right) d t=\int_{0}^{+\infty} e^{-t} d t<\infty
$$

so according to Theorem 1, all the agents converge to the consensus $x^{\infty}$ with the rate given by (30). Lastly, we compute explicitly:

$$
\dot{x}_{i}(t)=\frac{1}{M} \sum_{j=1}^{N} m_{j}(t)\left(x_{j}(t)-x_{i}(t)\right)=\left(\bar{X}(t)-x_{i}(t)\right), \quad i \in\{1, \cdots, N\},
$$

which gives: $\dot{x}_{i}(t)-\dot{x}_{j}(t)=-\left(x_{i}(t)-x_{j}(t)\right)$ and (31) follows.

Remark 5. For the classical weightless opinion dynamics (1) with constant interaction function $a \equiv 1$, the system converges exponentially to consensus at the value $\bar{x}$ with the same rate:

$$
\forall i \in\{1, \cdots, N\}, \quad x_{i}(t)=\bar{x}+e^{-t}\left(x_{i}^{0}-\bar{x}\right) .
$$

This is to be expected since the classical dynamics (1) are a special case of system (3)-(4)-(5) with equal and constant weights.

We now further study the evolution of the weight distribution, and exhibit fundamental differences in the behaviors of the three models (M1), (M2) and (M3).

Proposition 15. Consider Model (M1), with constant interaction function a $\equiv 1$. Suppose that for all $i \in\{1, \cdots, N\}, m_{i}(0)=m_{i}^{0}>0$. Then there exist $\left(\mu_{i}\right)_{i \in\{1, \cdots, N\}}$ positive constants such that for all $t \geq 0$ for all $i \in\{1, \cdots, N\}$ the weights satisfy: $m_{i}(t) \geq \mu_{i}>$ 0 . 
Proof. From (M1), the weights satisfy for all $i \in\{1, \cdots, N\}$ :

$$
\dot{m}_{i}=\frac{m_{i}}{M} \sum_{j=1}^{N} m_{j}\left\langle\frac{\dot{x}_{i}+\dot{x}_{j}}{2}, \frac{x_{i}-x_{j}}{\left\|x_{i}-x_{j}\right\|}\right\rangle \text {. }
$$

From Proposition 14,

$$
\left\|\frac{\dot{x}_{i}+\dot{x}_{j}}{2}\right\|=\left\|\bar{X}-\frac{x_{i}+x_{j}}{2}\right\| \leq D(t) \leq D(0) e^{-t} .
$$

From this we get:

$$
\dot{m}_{i} \geq-\frac{m_{i}}{M} \sum_{j=1}^{N} m_{j}\left\|\frac{\dot{x}_{i}+\dot{x}_{j}}{2}\right\| \geq-\frac{m_{i}}{M} \sum_{j=1}^{N} m_{j} D(0) e^{-t}=-m_{i} e^{-t} D(0) .
$$

Integrating with respect to time yields:

$$
\ln \left(\frac{m_{i}(t)}{m_{i}^{0}}\right) \geq-\int_{0}^{t} e^{-s} D(0) d s
$$

from which we obtain:

$$
m_{i}(t) \geq m_{i}^{0} e^{D(0)\left(e^{-t}-1\right)} \geq m_{i}^{0} e^{-D(0)}>0 .
$$

This result shows that in the case of Model (M1), there is no saturation behavior: if the weights are initially all positive, they remain bounded away from zero at all time, which implies that they remain well-distributed among the agents. This is illustrated in the numerical simulations presented in Figures 2 and 6.

We now show that we have the opposite behavior in the case of Model (M2).

Proposition 16. Consider Model (M2) with the heterophilious interaction $a \equiv 1$. Let $p_{i}^{0}=p_{i}(0)=\sum_{j=1}^{N}\left\|x_{i}^{0}-x_{j}^{0}\right\|$. Suppose that for all $(i, j) \in\{1, \cdots, N\}^{2}, p_{i}^{0} \neq p_{j}^{0}$, and let $k:=\operatorname{argmax}_{i \in\{1, \cdots, N\}} p_{i}^{0}$. Then

$$
\lim _{t \rightarrow \infty} m_{k}(t)=M \quad \text { and } \quad \forall i \neq k, \lim _{t \rightarrow \infty} m_{i}(t)=0 .
$$

Proof. From Proposition 14, for all $i \in\{1, \cdots, N\}$ :

$$
p_{i}(t)=\sum_{j=1}^{N}\left\|x_{i}(t)-x_{j}(t)\right\|=e^{-t} \sum_{j=1}^{N}\left\|x_{i}^{0}-x_{j}^{0}\right\|=e^{-t} p_{i}^{0} .
$$

Then from (M2),

$$
\dot{m}_{i}(t)=\frac{m_{i}(t)}{M}\left(\frac{p_{i}(t)}{\frac{1}{M} \sum_{j=1}^{N} m_{j}(t) p_{j}(t)}-1\right)=\frac{m_{i}(t)}{M}\left(\frac{p_{i}^{0}}{\frac{1}{M} \sum_{j=1}^{N} m_{j}(t) p_{j}^{0}}-1\right) .
$$


Let $k:=\operatorname{argmax}_{i \in\{1, \cdots, N\}} p_{i}^{0}$. Then $p_{k}^{0} \geq \frac{1}{M} \sum_{j=1}^{N} m_{j}(t) p_{j}^{0}$, so $\dot{m}_{k}(t) \geq 0$ for all $t \geq 0$. Since $m_{k}(t) \leq M$, there exists $\bar{m}_{k}$ with $0 \leq \bar{m}_{k} \leq M$ such that $\lim _{t \rightarrow \infty} m_{k}(t)=\bar{m}_{k}$ and $m_{k}(t) \leq \bar{m}_{k}$ for all $t \geq 0$. Suppose that $\bar{m}_{k}<M$. More specifically, suppose that there exists $\delta>0$ such that $\bar{m}_{k}=M-\delta$. There also exists $\varepsilon>0$ such that for all $i \neq k$, $p_{i}^{0} \leq p_{k}^{0}-\varepsilon$. We then write:

$$
\begin{aligned}
\frac{\frac{1}{M} \sum_{j=1}^{N} m_{j}(t) p_{j}^{0}}{p_{k}^{0}} & =\frac{1}{p_{k}^{0} M}\left(m_{k} p_{k}^{0}+\sum_{j \neq k} m_{j} p_{j}^{0}\right) \leq \frac{m_{k}}{M}+\frac{\left(M-m_{k}\right)\left(p_{k}^{0}-\varepsilon\right)}{M p_{k}^{0}} \\
& \leq \frac{m_{k}}{M}\left(1-\frac{p_{k}^{0}-\varepsilon}{p_{k}^{0}}\right)+\frac{p_{k}^{0}-\varepsilon}{p_{k}^{0}} \leq \frac{M-\delta}{M}\left(1-\frac{p_{k}^{0}-\varepsilon}{p_{k}^{0}}\right)+\frac{p_{k}^{0}-\varepsilon}{p_{k}^{0}} \\
& \leq 1-\frac{\delta}{M}\left(1-\frac{p_{k}^{0}-\varepsilon}{p_{k}^{0}}\right)=1-\frac{\delta \varepsilon}{M p_{k}^{0}} .
\end{aligned}
$$

Let $C:=(\delta \varepsilon) /\left(M p_{k}^{0}\right)>0$. We choose $\varepsilon$ small enough that $C>1$. Then

$$
\frac{p_{k}^{0}}{\frac{1}{M} \sum_{j=1}^{N} m_{j}(t) p_{j}^{0}} \geq \frac{1}{1-C}=1+\frac{C}{1-C}>1,
$$

and $\dot{m}_{k}$ satisfies:

$$
\dot{m}_{k} \geq \frac{m_{k}}{M}\left(1+\frac{C}{1-C}-1\right)=\frac{m_{k}}{M} \frac{C}{1-C}
$$

By Gronwall's lemma, for all $t \geq 0, m_{k}(t) \geq m_{k}^{0} e^{\frac{C}{M(1-C)} t}$, which contradicts $m_{k}(t) \leq M$. Hence $\bar{m}_{k}=M$ and because $\sum_{i=1}^{N} m_{i}=M$, for all $i \neq k, \lim _{t \rightarrow \infty} m_{i}(t)=0$.

Hence in the case of Model (M2), the agent with the largest initial positional influence $p_{k}^{0}$ becomes the leader: its weight tends to the total mass of the system $M$, while the weights of all the other agents tend to 0. This is illustrated in Figures 3 and 7.

Models (M3) and (M4) exhibit a third behavior, different from those of Models (M1) and (M2): starting from random initial conditions, two agents gain influence while all the other weights go to zero. We prove that indeed the state $m=$ $\left(\frac{M}{2}, \frac{M}{2}, 0, \ldots, 0\right)$ is an equilibrium for the weights.

Proposition 17. In models (M3) and (M4), any state satisfying

$$
\left\{\begin{array}{l}
m_{I}=m_{J}=\frac{M}{2} \\
m_{i}=0 \quad \text { for all } i \neq I, J
\end{array}\right.
$$

for any indices $I \neq J$, is an equilibrium for the weight dynamics, in the sense that $\dot{m}_{i}=0$ for all $i \in\{1, \cdots, N\}$.

Proof. Without loss of generality, we reorder the agents so that $I=1$ and $J=2$. For Model (M3), notice that for all $i \in\{3, \ldots, N\}, \dot{m}_{i}=0$. Furthermore, $I_{1}=\{2\}$ and $I_{2}=\{1\}$, so from (M3),

$$
\left\{\begin{array}{l}
\dot{m}_{1}=m_{1}\left(\frac{m_{2}}{m_{1}}-1\right)=m_{2}-m_{1}=0 \\
\dot{m}_{2}=m_{2}\left(\frac{m_{1}}{m_{2}}-1\right)=m_{1}-m_{2}=0
\end{array}\right.
$$


For Model (M4), again $\dot{m}_{i}=0$ for all $i \in\{3, \ldots, N\}$. The agents with non-zero masses satisfy:

$$
\dot{m}_{1}=m_{1}\left(\frac{m_{2}}{M} \frac{m_{1} a\left(\left\|x_{1}-x_{2}\right\|\right)\left\|x_{1}-x_{2}\right\|}{\frac{m_{1}^{2}}{M} a\left(\left\|x_{1}-x_{2}\right\|\right)\left\|x_{1}-x_{2}\right\|}-1\right)=m_{2}-m_{1}=0,
$$

and symmetrically, $\dot{m}_{2}=0$.

This is illustrated in Figures 4 and 8.

\section{Numerical simulations}

\subsection{Behavior of the four specific models with constant interaction function}

We start by illustrating the behavior of each of the four models for $d=1$. Figures 2, 3, 4 and 5 show the evolution of a group of 10 agents in $\mathbb{R}$, with the same initial conditions. The interaction function was chosen to be $a \equiv 1$. Notice that in all cases, the order of the opinions is preserved, as shown in Proposition 1. It is not the case for the order of the weights. As shown in Section 2.4, in all cases, the system converges to consensus asymptotically. In each of the figures 2 to 9 , the plot on the left represents the evolution of the opinions, each represented by a different color. The width of each curve is proportional to the agent's weight. For the one-dimensional plots (i.e. Figures 2 to 5), the dotted line represents the average position and the dashed line represents the weighted barycenter. The plot on the right shows the evolution of the corresponding weights, with the dashed line representing the average weight (constant by Proposition 2). Figure 2 illustrates the behavior of Model (M1) proven in Proposition 15: the weights vary little and stay bounded away from 0 . Figure 3 illustrates the behavior of Model (M2) proven in Proposition 16: one agent eventually gains the total mass of the system, and becomes leader. Notice that consensus is achieved at a different value than in Figure 2, due to the fact that one agent drags the group towards itself. Figures 4 and 5 illustrate the behaviors of Models (M3) and (M4), proven in Proposition 17: two agents eventually share equally the total mass of the system, and become co-leaders. Interestingly, with the same initial conditions, the two leaders are different in Model (M3) and Model (M4).

Figures $6,7,8$ and 9 show the evolution of a group of 10 agents in $\mathbb{R}^{2}$, with the same initial conditions. The interaction function was also chosen to be $a \equiv 1$. Notice that with Model (M2), consensus is reached before the leader gains the total mass of the system (Figure 7). Hence it does not have time to influence the position at which consensus is achieved. As in 1D, with Models (M3) and (M4), two agents become co-leaders, but they are different for each of the models (see Figures 8 and 9). 


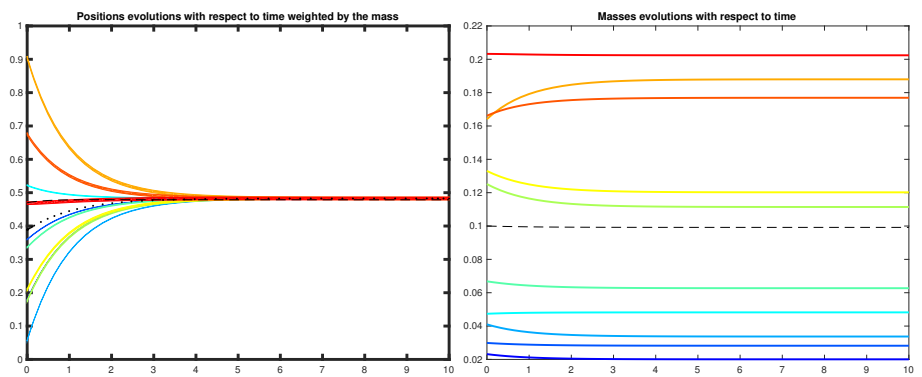

Figure 2: Evolution of opinions (left) and weights (right) of 10 agents in $\mathbb{R}$ with (M1).
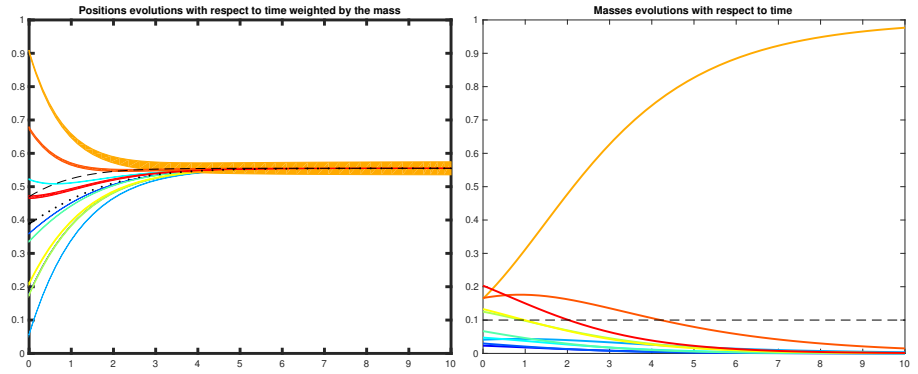

Figure 3: Evolution of opinions (left) and weights (right) of 10 agents in $\mathbb{R}$ with (M2).

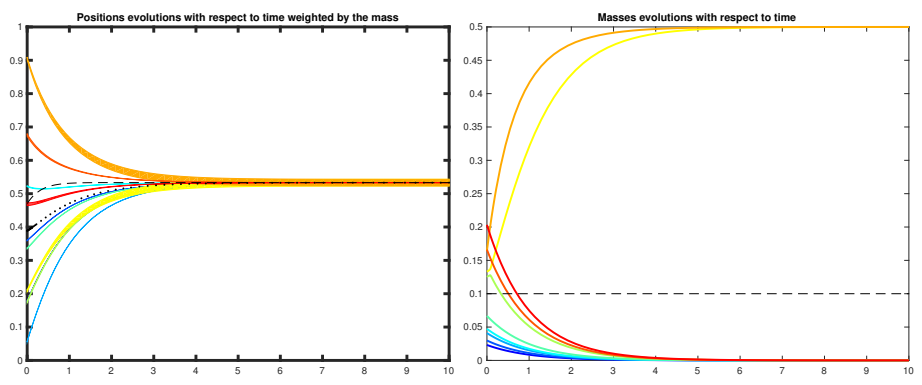

Figure 4: Evolution of opinions (left) and weights (right) of 10 agents in $\mathbb{R}$ with (M3).

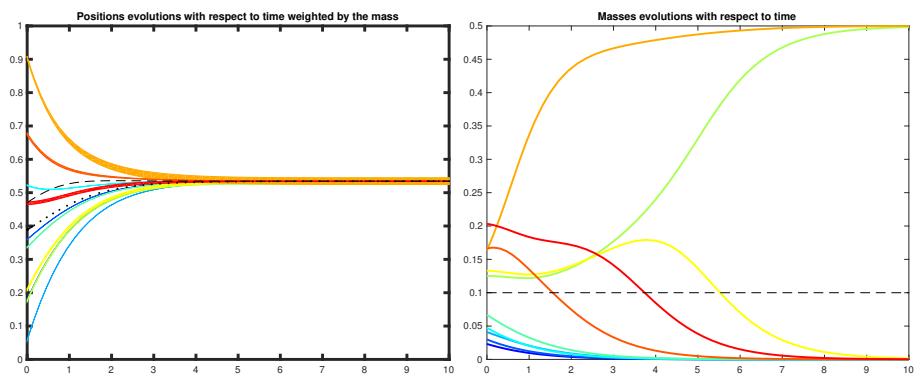

Figure 5: Evolution of opinions (left) and weights (right) of 10 agents in $\mathbb{R}$ with (M4). 

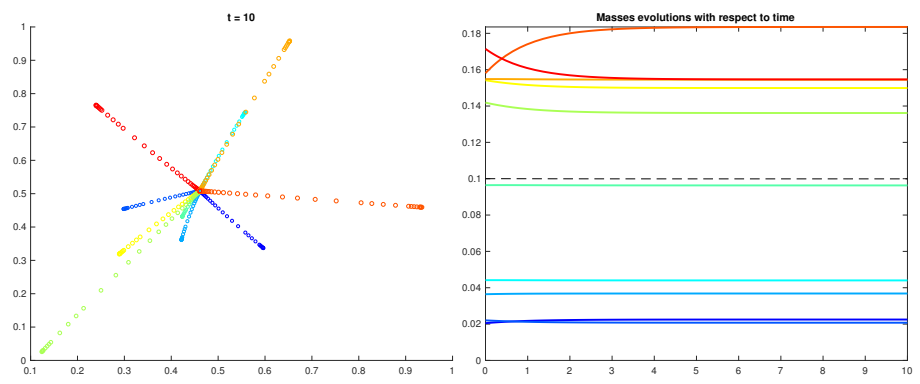

Figure 6: Evolution of opinions (left) and weights (right) of 10 agents in $\mathbb{R}^{2}$ with (M1).
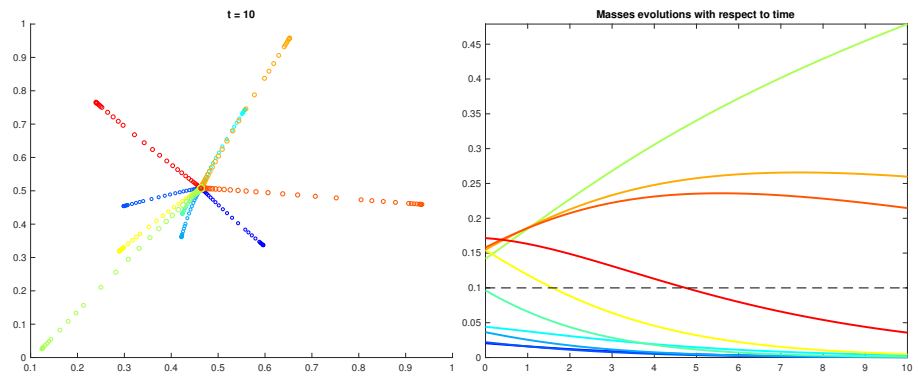

Figure 7: Evolution of opinions (left) and weights (right) of 10 agents in $\mathbb{R}^{2}$ with (M2).
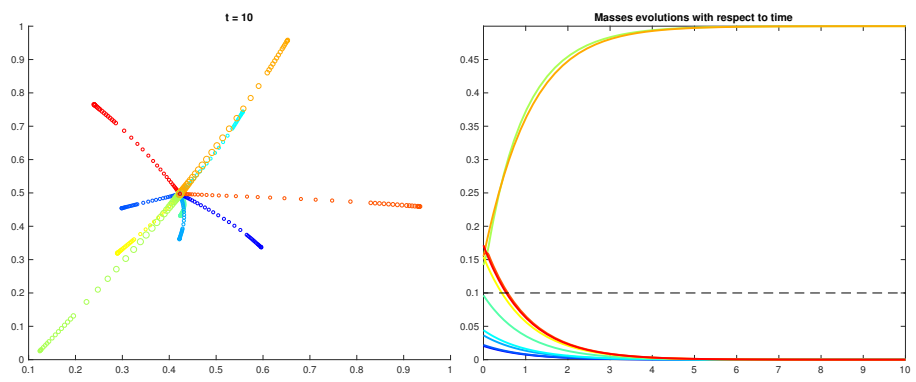

Figure 8: Evolution of opinions (left) and weights (right) of 10 agents in $\mathbb{R}^{2}$ with (M3).

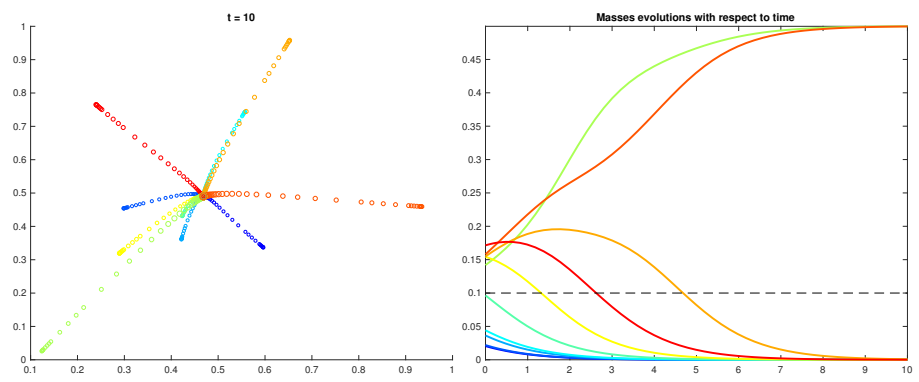

Figure 9: Evolution of opinions (left) and weights (right) of 10 agents in $\mathbb{R}^{2}$ with (M4). 


\subsection{Behavior of selected models with bounded confidence}

We now provide simulations for an interaction function with bounded support. In Section 2.5, we showed that for a model with constant weights, the system forms clusters separated by at least the distance of the support of the interaction function. We also showed through simple examples that the same behavior cannot be expected if the weights are allowed to evolve in time, and more specifically, to vanish. We now show general simulations in 1D exhibiting the behavior of the system when $a$ satisfies Hypothesis 3 with $R=0.2$.

Figure 10 shows the evolution of the opinions and weights for Model (M2). We observe cluster formation, with clusters separated by a distance greater than $R$, the support of $a$. Interestingly, whereas the opinions quickly reach an equilibrium, the weights keep oscillating between clusters (in the opinions plots, the width of each curve is proportional to the agent's weight). This is due to the fact that the weight dynamics allow exchange of mass between clusters (the denominator in (M2) sums over all agents, regardless of the distances between opinions). The weighted barycenter is shown to oscillate (dashed line), whereas the average opinion converges to an equilibrium (dotted line).

Figure 11 shows the evolution of the opinions and weights for Model (M3). Recall that with a constant interaction function, the system was shown to converge to an equilibrium in which two agents share equally the total mass of the system. This behavior is observed again here, but now at the level of each individual cluster. Figure 11 shows a situation where the system converges to three clusters. The one that converge at position $x=0.84$ is composed of three agents, but its mass is only shared between the green and the yellow agents, as shown by the weights' evolution. Similarly, the second cluster (at $x=0.4$ ) is composed of five agents, but only the green and the orange agents gain the total mass of the cluster. Lastly, the third cluster is only composed of two agents (red and blue) that share the mass equally.
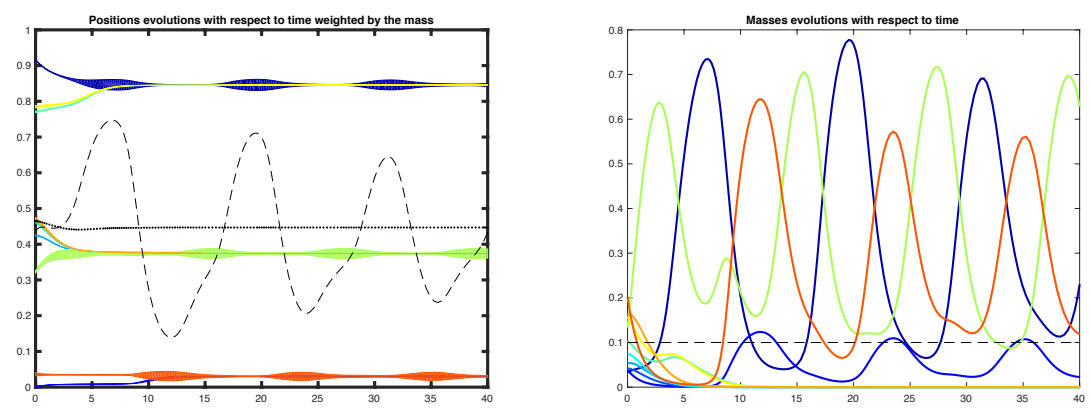

Figure 10: Evolution of opinions (left) and weights (right) of 10 agents in $\mathbb{R}$ with (M2), $a$ satisfying Hyp. 3 with $R=0.2$. 

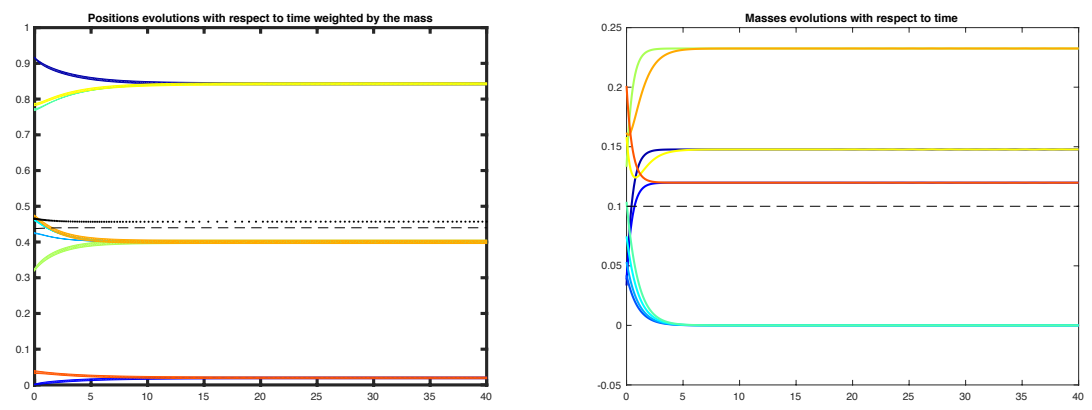

Figure 11: Evolution of opinions (left) and weights (right) of 10 agents in $\mathbb{R}$ with (M3), $a$ satisfying Hyp. 3 with $R=0.2$.

\subsection{Speed of convergence}

In this section, we compare the speed of convergence to consensus in each of the four models. We define the "dispersion" over time by:

$$
\Delta(t):=\frac{1}{N^{2}} \sum_{i, j=1}^{N}\left\|x_{i}(t)-x_{j}(t)\right\|^{2} .
$$

Simulations were run with $N=30$ agents and $d=3$ to test the effect of mass dynamics on speed of convergence to consensus. We used the interaction function $a(\cdot) \equiv 1$. Recall that the system converges asymptotically to consensus, as seen in Propositions 10 and 14 . We fix a final time $T=10$ and we consider that the system has "reached consensus" if $\Delta(T)<\varepsilon=10^{-6}$. We denote by $\omega$ the number of times that consensus was reached out of 100 simulations in 3 dimensions.

Two series of simulations were run. In the first one, whose results are shown in Table 1, all 30 initial weights were sampled randomly from the uniform distribution in $[0,1]$, and then normalized so that $M=\sum_{i=1}^{30} m_{i}=1$. The 30 initial positions were sampled randomly from the uniform distribution in $[0,1]^{3}$.

In the second series, whose results are shown in Table 2, all 30 initial weights were set to be equal to $m_{i}=1 / 30$, thus still satisfying $M=1$. The 30 initial positions were sampled randomly from the uniform distribution in $[0,1]^{3}$.

We denote by $\tau$ the average time to consensus given that the system achieved it. Additionally, $\bar{\Delta}(10)$ represents the mean dispersion at the end of the simulation $(T=$ 10) for all 100 simulations. As a benchmark for time to convergence, "Model NMD" shows statistics for the model with no mass dynamics. For Model (M1) the time to consensus is very similar to Model-NMD. This is expected because Model (M1) shows only weak mass dynamics in the sense that masses do not change dramatically from their initial values, see also simulations shown in Figures 2 and 6. Model (M2) shows 


\begin{tabular}{l|c|c|c|} 
Random initial mass & $\omega$ & $\tau$ & $\bar{\Delta}(10)$ \\
\hline M1 & 100 & 6.6580 & $3.2003 \times 10^{-8}$ \\
M2 & 100 & 6.8082 & $3.5569 \times 10^{-8}$ \\
M3 & 100 & 6.5051 & $2.7298 \times 10^{-8}$ \\
M4 & 100 & 6.4685 & $2.6024 \times 10^{-8}$ \\
Model-NMD & 100 & 6.6801 & $3.2298 \times 10^{-8}$
\end{tabular}

Table 1: Speed of convergence with randomly generated weights

\begin{tabular}{l|c|c|c|} 
Equal initial mass & $\omega$ & $\tau$ & $\bar{\Delta}(10)$ \\
\hline M1 & 100 & 6.6996 & $3.2798 \times 10^{-8}$ \\
M2 & 100 & 6.8559 & $3.7337 \times 10^{-8}$ \\
M3 & 100 & 6.6664 & $3.2040 \times 10^{-8}$ \\
M4 & 100 & 6.5616 & $2.8759 \times 10^{-8}$ \\
Model-NMD & 100 & 6.6886 & $3.2346 \times 10^{-8}$
\end{tabular}

Table 2: Speed of convergence with equal weights

greater average time to consensus. This suggests that having a leader who is the only one to have influence on the group is not optimal. Interestingly, Models (M3) and (M4) converge the fastest, which suggests that a system guided by two leaders converges more efficiently to consensus than a system guided by one unique leader, and than a system with no leader.

\subsection{Clustering of bounded confidence models with time-varying mass}

We now present simulations comparing the effect of each model's mass dynamics on the clustering behavior of the system. Ten simulations of each model (M1), (M2), (M3), (M4) and (M-NMD) were run with $N=30$ agents in three spatial dimensions $(d=3)$. As in Section 4.3, M-NMD refers to the model with no mass dynamics, i.e. $\Psi \equiv 0$. In order to observe clustering, we chose for these simulations an interaction function $a(\cdot)$ with bounded support, satisfying Hypothesis 3 with an interaction radius set to $R=0.4$. More specifically,

$$
\left\{\begin{array}{l}
a(r)=1 \text { for all } r \in(0, R) \\
a(r)=0 \text { for all } r \geq R .
\end{array}\right.
$$

As in Section 4.3, we first generated 10 sets of initial conditions (IC0 through IC9) with randomly generated opinions in $[0,1]^{3}$ and randomly generated masses in $[0,1]$, satisfying $M=\sum_{i=1}^{30} m_{i}=1$. The resulting number of clusters for each model is shown in Table 3, where the rightmost column shows for each model the mean number of clusters $\bar{c}$ of all 10 simulations. As expected, Model (M1) has a similar behavior to M-NMD because the mass dynamics are weak compared to those of (M2), (M3), and (M4). It is notable that M-NMD forms the least number of clusters on average. Mass 
seems to increase the speed at which nearby agents move toward another, and this would cause a cluster to form such that the less massive agents move toward more massive ones. These less massive agents would not have the attraction to draw the clusters together. An important note about (M3) and (M4) is that a high number of clusters arises. This can be explained by the fact that pairs of co-leading agents drain the masses of all the other ones, as shown in Figure 11. This affects clustering by leaving many singleton clusters of mass-less agents. Even if these agents are relatively close to each other (their distance being possibly less than the interaction radius $R$ ), they will have no attractive effect on any agent.

\begin{tabular}{l|rrrrrrrrrrr} 
& IC0 & IC1 & IC2 & IC3 & IC4 & IC5 & IC6 & IC7 & IC8 & IC9 & $\bar{c}$ \\
\hline M1 & 5 & 4 & 5 & 6 & 8 & 5 & 5 & 4 & 4 & 2 & 4.8 \\
M2 & 5 & 4 & 7 & 6 & 6 & 5 & 5 & 5 & 4 & 5 & 5.2 \\
M3 & 11 & 9 & 8 & 12 & 7 & 9 & 10 & 7 & 6 & 8 & 8.7 \\
M4 & 8 & 6 & 7 & 9 & 7 & 9 & 6 & 5 & 5 & 7 & 6.9 \\
M-NMD & 5 & 3 & 5 & 6 & 7 & 5 & 4 & 4 & 4 & 3 & 4.6
\end{tabular}

Table 3: Clustering comparison of the five models with randomly generated weights

For comparison, we ran a corresponding set of ten simulations (IP0 through IP9) with initial opinions chosen identically to those of simulations IC0 through IC9, but with initially equally distributed masses $m_{i}=\frac{1}{N}$ for all $i \in\{1, \cdots, N\}$. In this case, model M-NMD is exactly the classical HK model (1). The clustering results are displayed in Table 4. Several observations can be made by comparing the two tables. Firstly, as mentioned previously, the equal distribution of weights tends to reduce the number of clusters: for Models (M1), (M2), (M3) and M-NMD, the average number of clusters is lower with initial conditions IP0-IP9 than with initial conditions IC0-IC9. Moreover, observe that with initially equal masses, Model (M1) and Model M-NMD's performances are equivalent: even though they achieve different numbers of clusters for each simulation, their average is the same.

Comparing these results with those of Section 4.3, it is interesting to note that with a positive interaction function, Models (M3) and (M4) seem to achieve faster convergence to consensus, whereas in the case of bounded confidence, they result in greater clustering of the system. These first results might suggest ways to design efficient control strategies to either drive the system to consensus, or on the contrary to avoid clustering. 


\begin{tabular}{l|rrrrrrrrrrr} 
& IP0 & IP1 & IP2 & IP3 & IP4 & IP5 & IP6 & IP7 & IP8 & IP9 & $\bar{c}$ \\
\hline M1 & 5 & 5 & 5 & 6 & 3 & 5 & 3 & 3 & 4 & 3 & 4.2 \\
M2 & 5 & 5 & 6 & 5 & 4 & 5 & 3 & 5 & 5 & 4 & 4.7 \\
M3 & 10 & 6 & 6 & 11 & 7 & 8 & 8 & 6 & 5 & 9 & 7.6 \\
M4 & 10 & 6 & 7 & 12 & 8 & 7 & 5 & 7 & 4 & 6 & 7.2 \\
M-NMD & 5 & 5 & 5 & 6 & 3 & 4 & 3 & 3 & 5 & 3 & 4.2
\end{tabular}

Table 4: Clustering comparison of the five models with initially equal weights

\section{Conclusion and future directions}

In this paper we introduced an augmented model for first-order opinion dynamics by associating weights to each agent, and prescribing specific dynamics for the evolution of the weights. We showed that although the weights are allowed to vanish individually, convergence of the system to consensus still holds, as in the classical model, when the interaction function is uniformly positive on compact sets. We also showed that in the case of an interaction function with bounded support, convergence of the system to clustering still holds when the weights do not evolve in time. However, when we introduce weight dynamics, even if clustering can occur, we can no longer expect the clusters to form at distances necessarily greater than the support of the interaction function, as highlighted in simple examples. Numerical simulations suggest that the nature of the weight dynamics influences the rate at which the system converges to consensus or achieves clustering.

This work opens the way for future developments of opinion dynamics with timevarying weights. For instance, the weight dynamics could be seen as a control on the system, which one could use either to speed up or to impair convergence to consensus (see [24, 25]). Among other possible extensions, one could introduce a model in which the weights influence not only the amplitude of the interaction, but its range, which would be particularly significant if the interaction function had bounded support. Lastly, a natural future direction will be to establish the mean-field limit of the system, when the number of agents tends to infinity.

Acknowledgments: The authors acknowledge the support of the NSF project KI-Net, DMS Grant \#1107444. 


\section{References}

[1] A. Aydoğgu, M. Caponigro, S. McQuade, B. Piccoli, N. Pouradier Duteil, F. Rossi, and E. Trélat. Interaction network, state space and control in social dynamics. In N. Bellomo, P. Degond, and E. Tadmor, editors, Active Particles Volume 1, Theory, Methods, and Applications. Birkhauser-Springer, 2017.

[2] A. Aydoğdu, S. McQuade, and N. Pouradier Duteil. Opinion dynamics on Riemannian manifolds. Networks and Heterogeneous Media, 12(3), 2017.

[3] M. Ballerini, N. Cabibbo, R. Candelier, A. Cavagna, E. Cisbani, I. Giardina, V. Lecomte, A. Orlandi, G. Parisi, A. Procaccini, M. Viale, and V. Zdravkovic. Interaction ruling animal collective behavior depends on topological rather than metric distance: Evidence from a field study. Proceedings of the National Academy of Sciences, 105(4):1232-1237, 2008.

[4] N. Bellomo and A. Bellouquid. On the modeling of crowd dynamics: Looking at the beautiful shapes of swarms. Networks and Heterogeneous Media, 6:383-399, 2011.

[5] N. Bellomo and J. Soler. On the mathematical theory of the dynamics of swarms viewed as complex systems. Mathematical Models and Methods in Applied Sciences, 22(1140006), 2012.

[6] S. Camazine, J. Deneubourg, N. Franks, J. Sneyd, G. Theraulaz, and E. Bonabeau. Self organization in biological systems. Princeton University Press, 2003.

[7] M. Caponigro, A. C. Lai, and B. Piccoli. A nonlinear model of opinion formation on the sphere. Discrete and Continuous Dynamical Systems Ser. A, (9):42414268, 2015.

[8] I. Couzin, J. Krause, R. James, G. Ruxton, and N. Franks. Collective memory and spatial sorting in animal groups. J Theor Biol, 218(1-11), 2002.

[9] M. H. De Groot. Reaching a consensus. Journal of American Statistical Association, 69:118 - 121, 1974.

[10] J. C. Dittmer. Diskrete nichtlineare modelle der konsensbildung. Diploma thesis Universität Bremen, 2000.

[11] A. F. Filippov. Differential equations with discontinuous right-hand side, volume 51(93). Mat. Sb. (N.S.), 1960.

[12] J. R. P. French. A formal theory of social power. Psychological Review, 63:181 $-194,1956$.

[13] I. Giardina. Collective behavior in animal groups: theoretical models and empirical studies. Human Frontier Science Program Journal, (205-219), 2008.

[14] F. Harary. A criterion for unanimity in french's theory of social power. Cartwright D (Ed.), Studies in Social Power, 1959. 
[15] R. Hegselmann and A. Flache. Understanding complex social dynamics - a plea for cellular automata based modelling. Journal of Artificial Societies and Social Simulation, 1(3), 1998.

[16] R. Hegselmann and U. Krause. Opinion dynamics and bounded confidence models, analysis, and simulation. Journal of Artificial Societies and Social Simulation, 5(3), 2002.

[17] J. M. Hendrickx and J. N. Tsitsiklis. Convergence of type-symmetric and cutbalanced consensus seeking systems. IEEE Transactions on Automatic Control, 58(1):214-218, Jan 2013.

[18] P. Jabin and S. Motsch. Clustering and asymptotic behavior in opinion formation. Journal of Differential Equations, 257(11):4165-4187, 122014.

[19] J. Krause and G. Ruxton. Living in groups. Oxford series in ecology and evolution. Oxford University Press, New York, 2002.

[20] U. Krause. Soziale dynamiken mit vielen interakteuren, eine problemskizze. Krause U and Stöckler M (Eds.) Modellierung und Simulation von Dynamiken mit vielen interagierenden Akteuren, Universität Bremen, pages 37 - 51, 1997.

[21] U. Krause. A discrete nonlinear and non-autonomous model of consensus formation. Elaydi S, Ladas G, Popenda J and Rakowski J (Eds.), Communications in Difference Equations, Amsterdam: Gordon and Breach Publ., pages 227 - 236, 2000.

[22] K. Lehrer. Social consensus and rational agnoiology. Synthese, 31:141 - 160, 1975.

[23] S. Motsch and E. Tadmor. Heterophilious dynamics enhances consensus. SIAM Review, 56(4):577-621, 2014.

[24] B. Piccoli, N. Pouradier Duteil, and B. Scharf. Optimal control of a collective migration model. Mathematical Models and Methods in Applied Sciences, 26(02):338-417, 2015.

[25] B. Piccoli, N. Pouradier Duteil, and E. Trélat. Sparse control of HelgselmannKrause models: Black hole and declusterization. submitted, 2018.

[26] B. Piccoli and F. Rossi. Measure-theoretic models for crowd dynamics. Springer International Publishing, 2018. 